\title{
Computational and Experimental Characterization of the Mach 6 Facility Nozzle Flow for the Enhanced Injection and Mixing Project at NASA Langley Research Center
}

\author{
Tomasz G. Drozda, ${ }^{\dagger}$ Karen F. Cabell ${ }^{\dagger}$ Bradley J. Passe, ${ }^{\ddagger}$ and Robert A. Baurle, ${ }^{\S}$ \\ NASA Langley Research Center, Hampton, VA, 23681
}

\begin{abstract}
Computational fluid dynamics analyses and experimental data are presented for the Mach 6 facility nozzle used in the Arc-Heated Scramjet Test Facility for the Enhanced Injection and Mixing Project (EIMP). This project, conducted at the NASA Langley Research Center, aims to investigate supersonic combustion ramjet (scramjet) fuel injection and mixing physics relevant to flight Mach numbers greater than 8. The EIMP experiments use a two-dimensional Mach 6 facility nozzle to provide the high-speed air simulating the combustor entrance flow of a scramjet engine. Of interest are the physical extent and the thermodynamic properties of the core flow at the nozzle exit plane. The detailed characterization of this flow is obtained from three-dimensional, viscous, Reynolds-averaged simulations. Thermodynamic nonequilibrium effects are also investigated. The simulations are compared with the available experimental data, which includes wall static pressures as well as in-stream static pressure, pitot pressure and total temperature obtained via in-stream probes positioned just downstream of the nozzle exit plane.
\end{abstract}

\section{Introduction}

$\mathrm{T}$ HE Arc-Heated Scramjet Test Facility (AHSTF) ${ }^{1-3}$ is a high-enthalpy wind tunnel used for testing supersonic combustion ramjet (scramjet) engines. With recent upgrades to extend the low-Mach simulation capability, the facility can produce total enthalpies corresponding to flight Mach numbers of about 2.5 to 8 . The high total enthalpies are achieved by over-heating a portion of the total facility air with an electric arc and then mixing it with unheated bypass air to obtain the desired mixture total enthalpy. The combined air mixture is then expanded through a converging-diverging facility nozzle. Several facility nozzles with various exit Mach numbers are available with the nozzle selection depending on the desired flight simulation. The nozzle Mach number can be equal to the flight Mach number for a free-jet configuration, or less than the flight Mach number for a "semi-freejet" or "direct-connect" configuration, where some level of precompression is simulated.

The flow profiles at the exit of the facility nozzle depend on the nozzle geometry ${ }^{4}$ (e.g., axisymmetric, square or rectangular), as well as, to a lesser degree, the heat transfer to the nozzle walls. Furthermore, the surface contour of a facility nozzle is carefully designed for operation at a specific value of the total enthalpy and Mach number to prevent the formation of shock or expansion waves during the flow expansion process. However, because designing and fabricating a facility nozzle for each condition of interest is not always financially possible, the existing nozzles are often used at off-design conditions, or more specifically at total enthalpies that differ from that for which the nozzle was designed. In those situations, it is prudent to characterize the flow profile because both the physical extent and the aero- and thermodynamic properties of the nozzle core flow will change as a function of the total enthalpy.

The Enhanced Injection and Mixing Project (EIMP) ${ }^{5}$ being conducted at the NASA Langley Research Center is utilizes the AHSTF for experimental investigations of scramjet fuel injection and mixing processes. The EIMP aims to improve understanding of the fundamental physics that govern these processes, as well as, to develop concepts and functional relationships between mixing performance and losses relevant to flight Mach numbers greater than 8. The

\footnotetext{
${ }^{\dagger}$ Research Aerospace Engineer, Hypersonic Airbreathing Propulsion Branch, AIAA Senior Member.

$\ddagger$ Research Engineer, Analytical Mechanics Associates, Inc., AIAA Member.

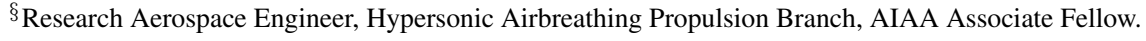


research approach is a closely-coupled experimental and computational effort, with experiments intended for exploring injection concepts and for anchoring computational fluid dynamics (CFD) simulations. The experiments utilize a Mach 6 facility nozzle, designed for Mach 7 total enthalpy, to produce the high-speed air simulating the combustor entrance flow of a scramjet engine. Although the combustor entrance Mach number matches that for flight, the maximum total enthalpy for these experiments is limited by the thermal-structural limit of the uncooled experimental hardware, and corresponds to a Mach 4.25 flight enthalpy. Because this total enthalpy is significantly lower than the Mach 7 design total enthalpy, some uncancelled waves and reduced exit "core" size might be expected. Additionally, detailed knowledge of the nozzle exit flow is necessary for accurate CFD simulations of the mixing flow fields. Therefore, computational simulations of the Mach 6 nozzle flow at the planned EIMP test conditions were performed and nozzle exit flow survey tests were conducted to gather data to validate the CFD. The computational simulations include the effects of thermal nonequilibrium, which can be present as a result of rapidly expanding the heated air containing rotationally and vibrationally excited species through a facility nozzle. ${ }^{6}$ It should be noted that previously reported pretest CFD simulations ${ }^{7}$ utilized the as-designed nozzle geometry, whereas the current work utilizes the as-built geometry, with the most significant difference being that the as-built throat area is $7 \%$ smaller than the as-designed nozzle throat area.

The paper is organized as follows: first, a description of the Mach 6 nozzle flow survey experiment is provided. This includes the descriptions of the facility and nozzle, and the test conditions and acquired data. Next, an overview of the CFD approach is given. Discussion of numerical considerations that apply to all simulations is presented, as well as, results illustrating the impact of several numerical modeling choices (grid resolution, turbulence model, thermal boundary condition, and thermal nonequilibrium) on the core flow. The CFD calculations are then compared with experimentally measured wall static pressures and in-stream nozzle-exit measurements of static pressure, pitot pressure and total temperature. The paper is then summarized, and future efforts are described.

\section{Mach 6 Nozzle Flow Survey Tests}

As described above, in the AHSTF, a portion of the total facility air is heated by an electric arc and then mixed with unheated air to obtain the desired mixture total enthalpy. The arc-heated "main" air exits the heater at a bulk temperature of about $4000^{\circ} \mathrm{R}$ and enters a plenum chamber, upstream of the nozzle, where the unheated "bypass" air is introduced. The bypass air is injected radially to break up the swirling motion of the main air and promote mixing of the two streams. This method of heating only a portion of the total air to elevated temperatures and then mixing it with cooler air to achieve the desired total enthalpy is necessary because the arc heater cannot operate in a stable mode while processing the full facility air flow rates. As a result of arc-heating, the air contains trace amounts of nitric oxide (NO) as reported by Cabell and Rock ${ }^{8}$. Considering this work, the mole fraction of NO in the test gas for the EIMP test conditions is estimated to be about $1 \%$.

Flow in the plenum chamber is subsonic and nearly stagnated, therefore, the stagnation pressure is well approximated by a plenum wall pressure measurement. Total mass flow rate is measured using calibrated sonic venturi flow meters in both the main and bypass air supply lines. Plenum stagnation enthalpy is calculated using a sonic throat enthalpy calculation, which accounts for caloric imperfections. ${ }^{9}$ Inputs to this calculation are the measured stagnation pressure, the measured total mass flow rate, the measured nozzle throat area, and an assumed nozzle discharge coefficient of one. This last assumption can be readily verified using the nozzle CFD, which reveals that for the EIMP test conditions, the error associated with this assumption is at most $0.7 \%$. The plenum stagnation temperature is then calculated from the stagnation enthalpy assuming thermally perfect air.

The facility nozzle used in the current experiments, referred to as the "Mach 6 2D" nozzle, was designed to produce a Mach 6 core flow with minimal nonuniformities at a Mach 7 flight enthalpy. ${ }^{4}$ A side view of the nozzle is shown in Fig. 1. The top and bottom walls are contoured, but the side walls are flat. The flow cross-section is 10 inches wide throughout with a height of 0.1 , and 10 inches at the throat and exit, respectively. The length of the expansion section from the throat to the exit plane is 70 inches. The nozzle throat section is made of copper, which is water cooled to allow for continuous run times. The expansion section, starting from about 12.5 inches downstream of the throat, is made from stainless steel. The nominal operating conditions used in the EIMP correspond to a plenum stagnation pressure of $625 \mathrm{psia}$ and a plenum stagnation temperature ranging from 1315 to $1760^{\circ} \mathrm{R}$. The range is determined by the condensation limit of expanding air on the low end, and by the thermal-structural limit of the uncooled experimental hardware located downstream of the nozzle on the high end. These values correspond to Mach 3.4 and 4.25 flight enthalpies, respectively, at a flight dynamic pressure of $1500 \mathrm{psf}$. The nominal, ideal expansion characteristics, based on the geometric area ratio, for the nozzle are summarized in Table 1. 


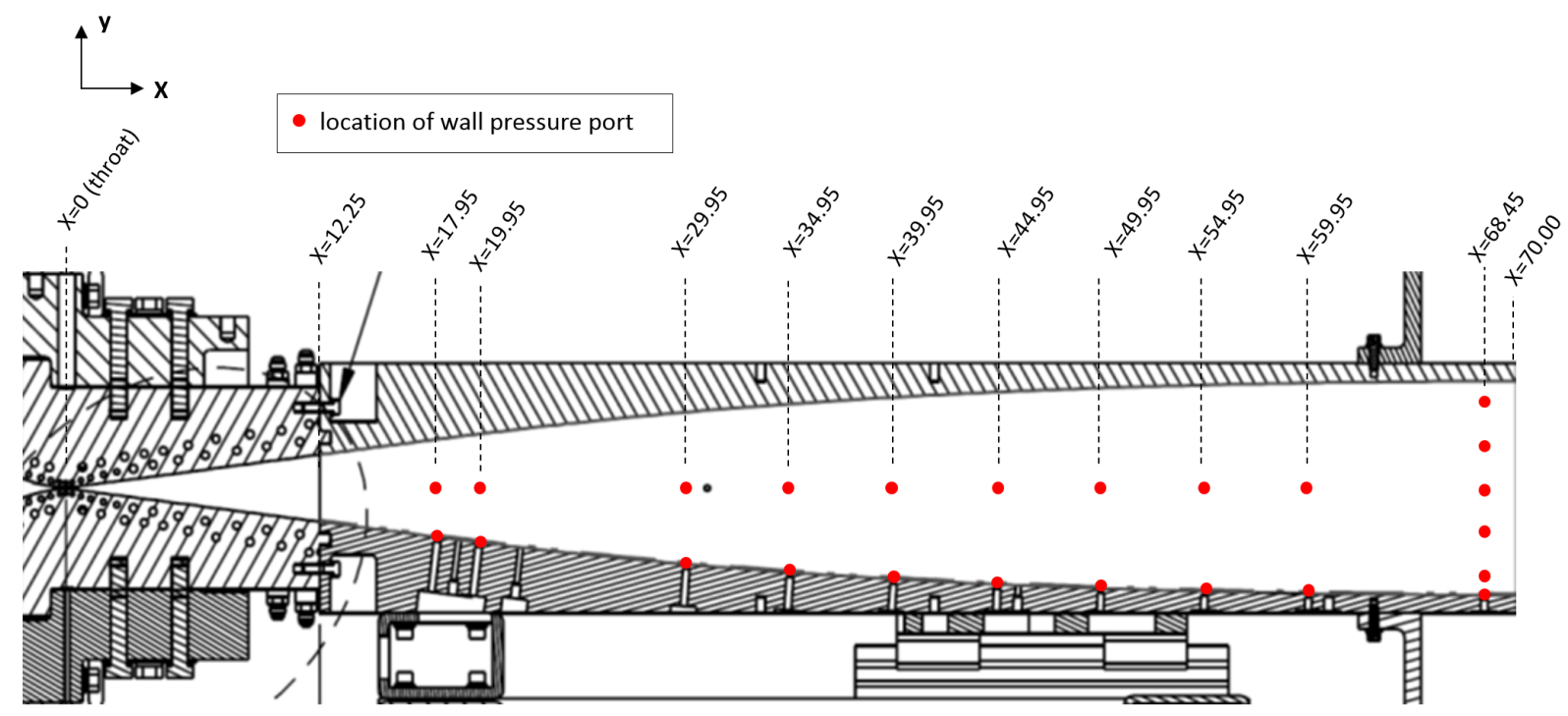

Figure 1. Cross-section of the Mach 6 facility nozzle (dimensions in inches).

As the facility air expands through the throat and into the supersonic portion of the long Mach 6 nozzle, fairly thick (up to about 2 inches) boundary layers develop along its walls. ${ }^{7}$ These boundary layers reduce the nozzle effective exit area, and thereby change the nozzle area ratio and the core flow exit flow properties. Previously reported pretest CFD simulations ${ }^{7}$ indicated that the resulting core flow Mach number is about 6.5. Furthermore, the turbulent boundary layers exhibit a reduction in the growth rate, under the favorable pressure gradient flow conditions as compared to the zero-pressure gradient flat plate case. This phenomena is important because it impacts the final thickness of the boundary layer and therefore, the nozzle core flow exit properties. Since most, if not all, of the turbulence models are calibrated using the zero-pressure-gradient flat plate experimental data, ${ }^{10}$ the predictions under adverse or favorable pressure gradients may not be optimal. Furthermore, since CFD is often used for nozzle design, any misprediction of the boundary layer character during the nozzle design phase has the potential to impact the actual Mach number of the nozzle, especially for high Mach number nozzles, which are generally much longer than their low Mach number counterparts, and therefore, allow the boundary layers to develop and grow over longer distances.

All four walls of the nozzle are instrumented with static pressure ports. The sidewalls are defined as right and left as viewed looking upstream. An axial row of static pressure ports exists along the centerline of both the bottom wall and the right sidewall at the locations indicated in Fig. 1. The left and top walls contain ports at a subset of these same axial locations. Additionally, at an axial station of $x=68.45$ inch ( 1.55 inches upstream of the nozzle exit plane), there

Table 1. Nominal plenum, and inviscid and isentropic expansion conditions for the Mach 6 facility nozzle.

\begin{tabular}{c|c|c} 
Property & Low Tot. Temp. & High Tot. Temp. \\
\hline Total Pressure $(\mathrm{psia})$ & 625 & 625 \\
Total Temperature $\left({ }^{\circ} \mathrm{R}\right)$ & 1315 & 1760 \\
Mach & 6.93 & 6.87 \\
$\dot{m}(\mathrm{lbm} / \mathrm{s})$ & 8.92 & 7.66 \\
Density $\left(\mathrm{lbm} / \mathrm{ft}^{3} \times 10^{-3}\right)$ & 3.345 & 2.459 \\
Pressure $(\mathrm{psia})$ & 0.1574 & 0.1608 \\
Temperature $\left({ }^{\circ} \mathrm{R}\right)$ & 126.6 & 175.9 \\
$\mathrm{U}(\mathrm{ft} / \mathrm{s})$ & 3828 & 4473 \\
Re/L $(1 / \mathrm{in})$ & $238 \mathrm{e} 3$ & $160 \mathrm{e} 3$ \\
Area Ratio & 102.04 & 102.04
\end{tabular}




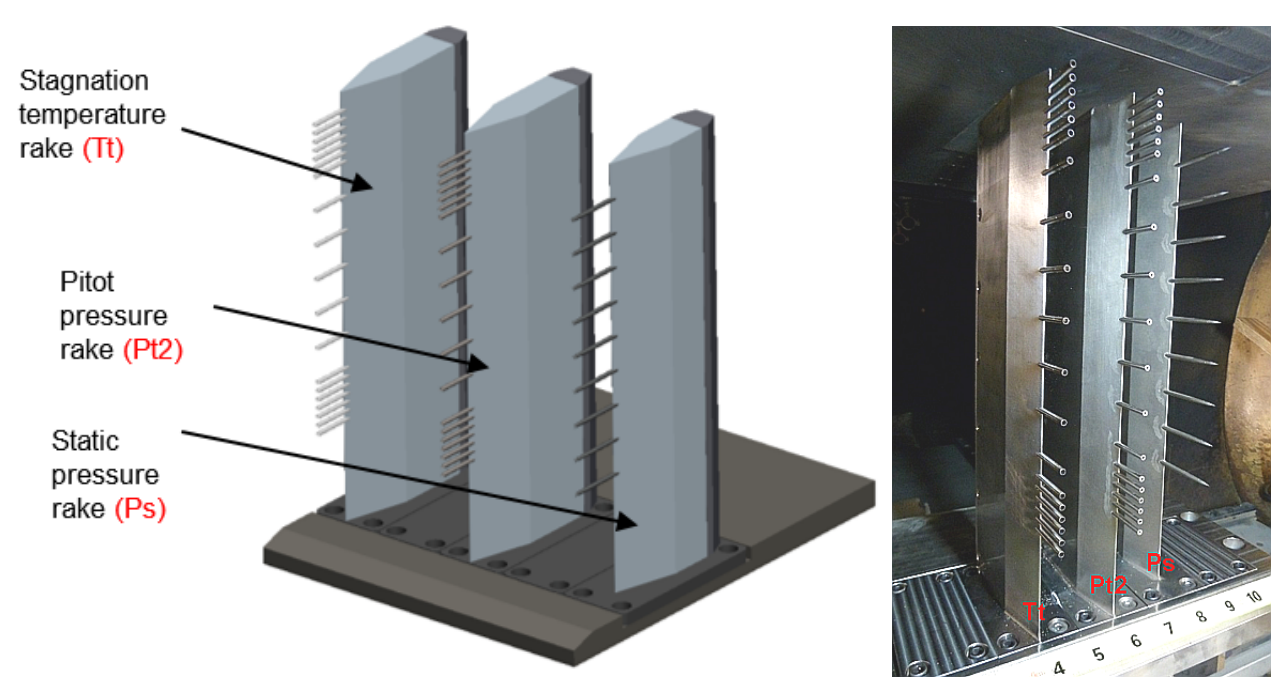

Figure 2. A CAD image and a photograph of the in-stream rake assembly. From left-to-right: total temperature, pitot pressure, and static pressure rakes.

are five ports on each wall, distributed spanwise (on the top and bottom walls) or vertically (on the sidewalls).

Measurements in the core flow of the nozzle exit were obtained by an in-stream rake system consisting of three rakes mounted on a pallet: a total temperature $\left(T_{t}\right)$ rake, a pitot pressure $\left(P_{t 2}\right)$ rake, and a static pressure $\left(P_{s}\right)$ rake. An isometric view of the rake assembly, as well as, a photograph are shown in Fig. 2. Spanwise spacing between rakes is fixed at two inches. All probe tips lie in a common axial plane, which is located 0.050 inches downstream of the nozzle exit plane. The rake assembly was translated to three different spanwise positions to provide data for comparisons with the CFD simulations.

Vertical spacing between the probes for each rake is shown in Fig. 3. The pitot pressure probes are constant diameter tubes having 0.125 -inch outer diameter and 0.035 -inch wall thickness. The stagnation temperature probes are 0.125-inch outer diameter tubes having 0.010-inch wall thickness and an exposed thermocouple bead recessed 0.25 inches inside the tube. They are the same design used in previous flow surveys of other AHSTF nozzles, ${ }^{11}$ with the exception that the tube material and thermocouple type (type K) were selected for the lower temperature range associated with the EIMP conditions. The static pressure probe geometry, shown in Fig. 4, is based on the design by Pinckney ${ }^{12}$. However, this particular probe geometry was designed for a lower freestream Mach number and intended for flow surveys of the Mach 2.2 and 3.5 facility nozzles. Probe surface pressure distributions from axisymmetric CFD simulations assuming thermally equilibrated flow over the probe at freestream Mach numbers of 2.2, 3.5 and 6.35 are shown in Fig. 5. These results are used to derive a probe recovery factor, which is a ratio of measured to freestream static pressure, as a function of freestream Mach number to be applied to the measurements. The core flow conditions for the Mach 6.35 flow for the static pressure probe simulations were obtained from the previous Mach 6 nozzle simulations of Drozda et al. ${ }^{7}$ utilizing the as-designed nozzle geometry and the high total temperature conditions. The values of the recovery factors for the current experiments are 1.29 for the core, and 1.08 for the top- and bottom-most static pressure probes. A lower value is used for the latter, because these probes retrieve measurements from the top and bottom nozzle wall boundary layers, where the Mach number is estimated to be about 4.7. Additional simulations showed that the recovery factor for the core flow would be about $3 \%$ lower for the low than high total temperature conditions, and about $1 \%$ lower if thermal nonequilibrium effects were considered.

All nozzle wall and in-stream pressure data were acquired at $12 \mathrm{~Hz}$ with an electronic scanning pressure (ESP) system. All other experimental data were acquired at $50 \mathrm{~Hz}$. The measurement uncertainty for the nozzle wall pressures upstream of the $x=68.45$ inches station and for the pitot pressures is \pm 0.015 psi. The measurement uncertainty for the nozzle wall pressures at the $\mathrm{x}=68.45$-inch station and for the in-stream probe static pressures is $\pm 0.0075 \mathrm{psi}$. The measurement uncertainty for the probe total temperatures is estimated to be $\pm 4.7^{\circ} \mathrm{R}$. All data were time-averaged over a six second period of "steady" conditions. The time-averaged values of facility stagnation pressures and temperature can vary run-to-run from their target nominal values (listed in Table 1). These variations can be up to about $2 \%$, and $3 \%$ for the stagnation pressure and stagnation temperature, respectively. For the purpose of comparison of the 

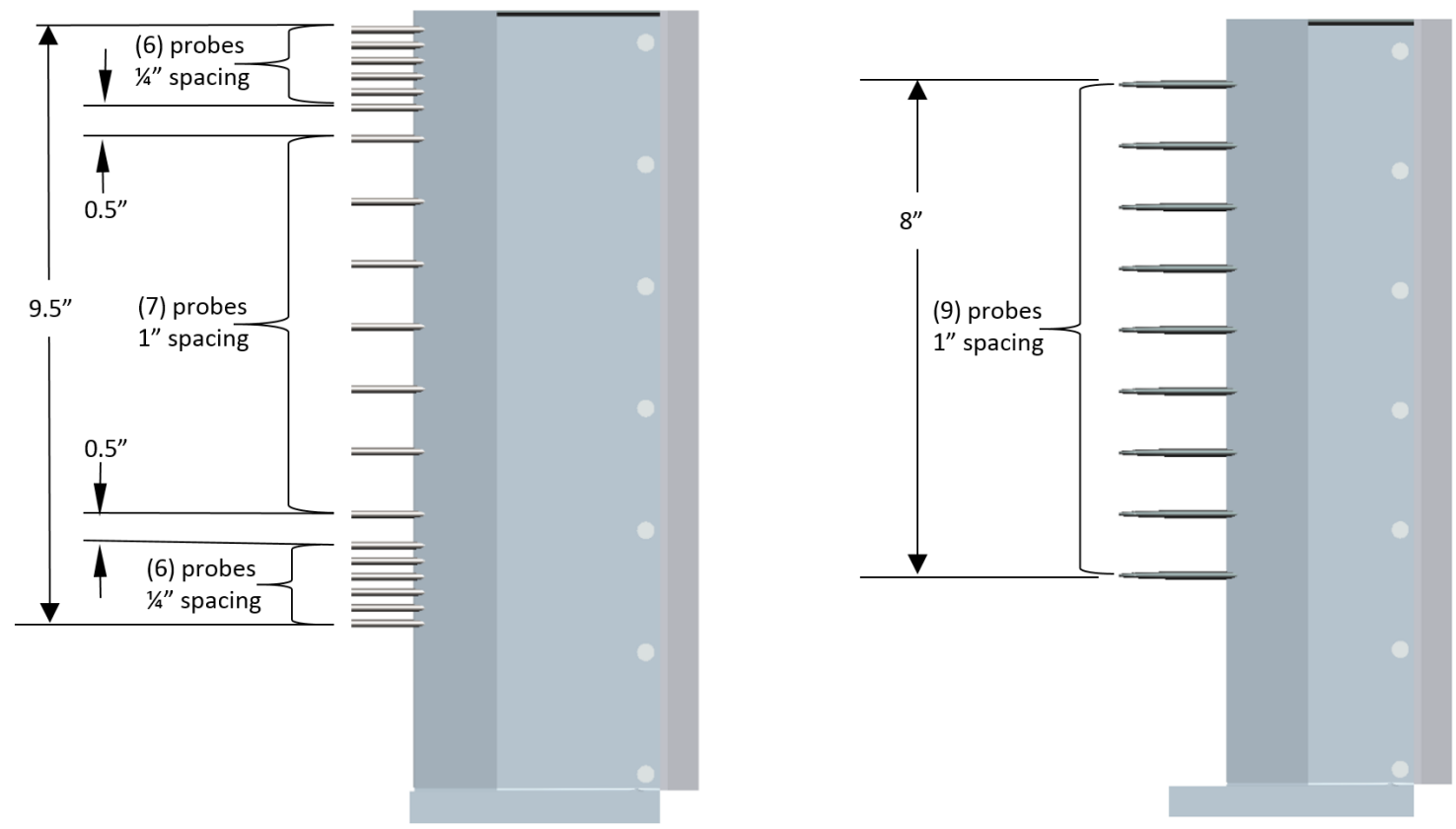

Stagnation pressure and stagnation temperature rake

Static pressure rake

Figure 3. Side views of the static and pitot pressure rakes, and the total temperature rake showing vertical probe spacing (dimensions in inches).
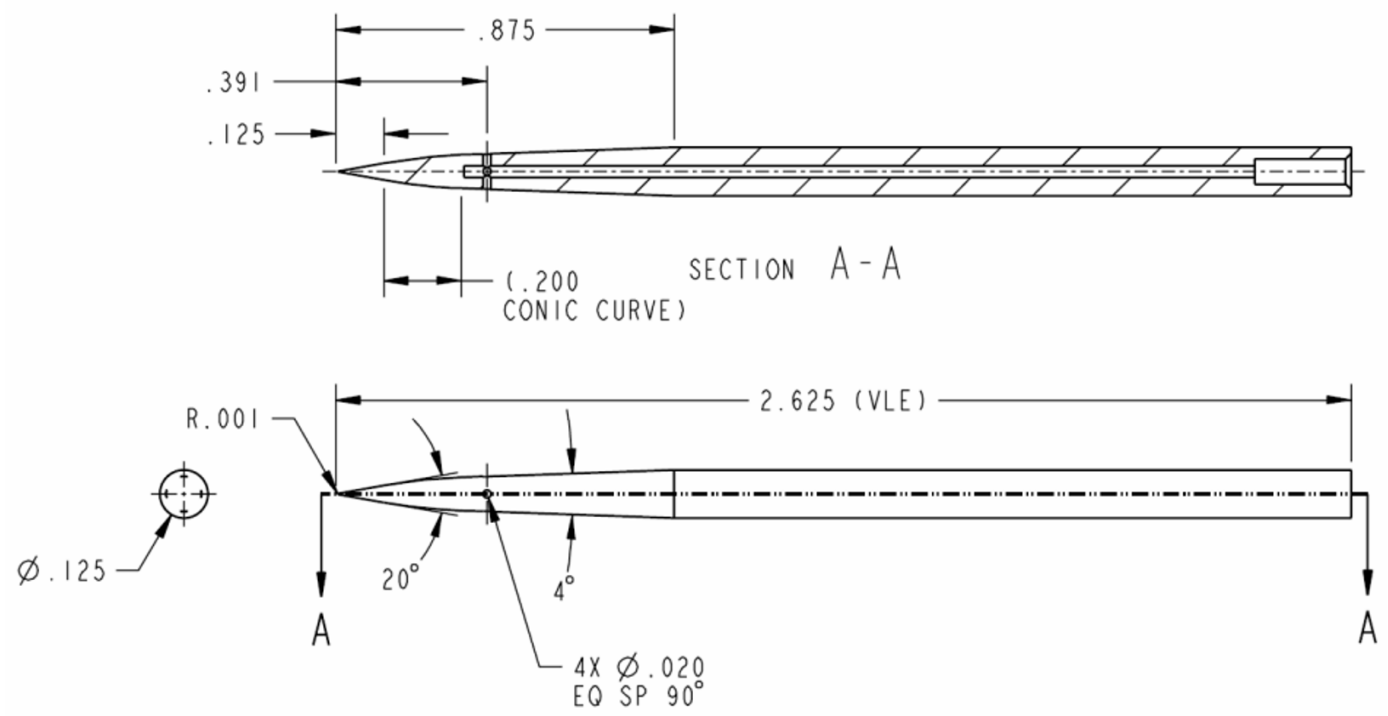

Figure 4. Static pressure probe geometry (dimensions in inches). 


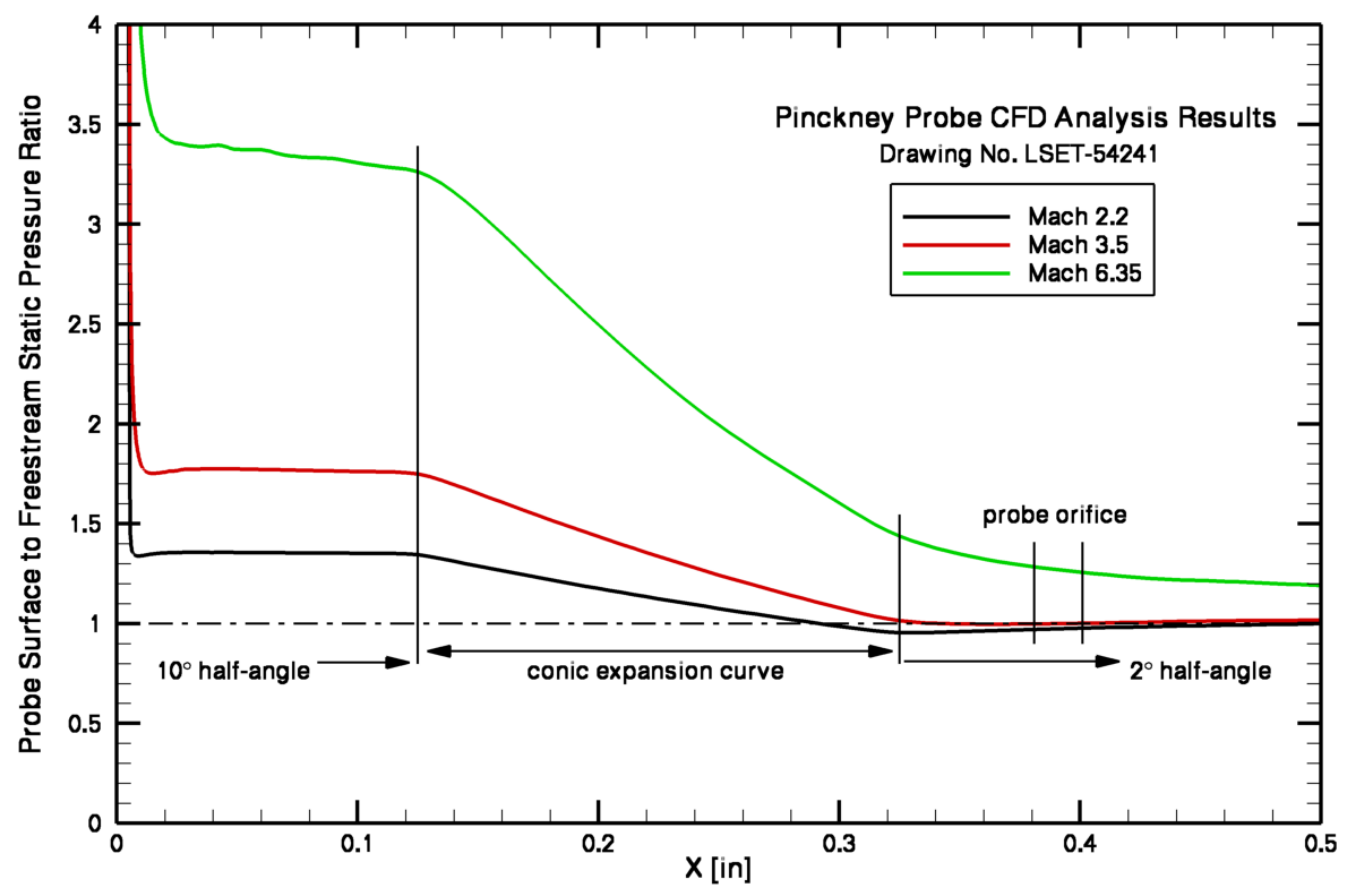

Figure 5. Static pressure probe surface distribution for freestream Mach numbers of 2.2, 3.5 and 6.35 
experimental data to the CFD, all the time-averaged wall and probe pressure data are rescaled by a ratio of the target and time-averaged facility stagnation pressure and all the time-averaged probe total temperature data are rescaled by a ratio of the target and time-averaged facility stagnation temperatures. Additionally, a recovery factor is applied to the static pressure probe measurement, as described above, depending on the probe location. Using the results of the CFD simulations discussed next, the predicted Mach number in the core flow was used to calculate the recovery factor. No corrections were applied to the total temperature probe measurements to account for radiation heat loss because the previous nozzle flow surveys using this probe design ${ }^{11}$ demonstrated a recovery factor of one for even higher total enthalpy flow conditions (i.e., Mach 5).

\section{Numerical Simulations and Sensitivities}

The numerical simulations were performed using the Viscous Upwind aLgorithm for Complex flow ANalysis (VULCAN-CFD) code. ${ }^{13}$ VULCAN-CFD is a multi-block; hybrid structured- and unstructured-grid, cell-centered, finite-volume solver widely used for all-speed flow simulations. For this work, Reynolds-averaged simulations (RAS) were performed. The advective terms were computed using a MUSCL scheme ${ }^{14}$ with the Low-Dissipation Flux-Split Scheme (LDFSS) of Edwards ${ }^{15}$. A nonreacting, thermally perfect mixture of $21 \%$ oxygen $\left(\mathrm{O}_{2}\right), 78 \%$ nitrogen $\left(\mathrm{N}_{2}\right)$, and $1 \% \mathrm{NO}$ was used for the facility air. A small amount of $\mathrm{NO}$ was present to account for production of this species in the arc-heater of the experimental facility. ${ }^{8}$ The thermodynamic properties of mixture components were computed using the curve fits of McBride et al. ${ }^{16}$. For calculations utilizing the thermodynamic nonequilibrium model, ${ }^{17}$ these curve fits are evaluated at the vibrational temperature. ${ }^{6}$ The governing equations were integrated using an implicit diagonalized approximate factorization (DAF) method ${ }^{18}$ and, for some cases, incomplete LU (ILU) factorization. ${ }^{19}$ The current work used the baseline turbulence model of $\mathrm{Mente}^{20}$, although some results utilizing an explicit algebraic Reynolds-stress model (EARSM) of Rumsey and Gatski ${ }^{21}$ are also shown to assess turbulence model sensitivity. The Reynolds heat and species mass fluxes were modeled using a gradient diffusion model with turbulent Prandtl and Schmidt numbers of 0.9 and 0.5 , respectively. The Wilcox wall matching functions ${ }^{10}$ were also used, where appropriate, to reduce the near-wall grid resolution requirements. However, integrate-to-the wall simulations were also performed to assess the impact of near-wall modeling on the boundary layer thickness. A grid sensitivity study was performed for several simulations with negligible changes in flow properties being computed when the fine grid resolution was reduced by a factor of two in each direction. All simulations were converged until the total integrated mass flow rate and the total integrated heat flux on the walls remained constant to about six significant digits. This typically occurred when the value of the $\mathrm{L}_{2}$-norm of the steady-state equation-set residual decreased by about 4-5 orders of magnitude.

Subsonic inflow boundary conditions specifying a total pressure of 625 psia and temperature of $1760^{\circ} \mathrm{R}$ were used at the inflow located upstream of the nozzle throat for the high enthalpy case. For the low enthalpy case, a total pressure of $625 \mathrm{psia}$ and temperature of $1315^{\circ} \mathrm{R}$ were used. First-order extrapolation was used at the supersonic outflow of the nozzle. The walls were modeled using a no-slip boundary condition for the velocities. The temperatures at the nozzle walls were obtained by locally solving a one-dimensional (1D), steady-state heat conduction equation with the experimentally-obtained average values of the nozzle outer-wall temperatures as an input. However, isothermal wall temperature boundary conditions were also included in this study to evaluate the sensitivity of the boundary layer to the near-wall thermal environment. The pitot pressure at the nozzle exit plane was computed iteratively using the Rankine-Hugoniot jump conditions with the flow assumed to be vibrationally frozen through the normal shock jump (for simulations that included thermal nonequilibrium modeling) and then thermally equilibrated to stagnation. The simulations were initialized using a solution of the corresponding 1D inviscid, isentropic and adiabatic solution to a converging-diverging nozzle problem.

Taking advantage of the nozzle symmetries, only the top-left quarter of the full nozzle geometry was simulated. The $\mathrm{x}, \mathrm{y}$, and $\mathrm{z}$, coordinates are aligned in the streamwise, cross-stream (pointing toward the nozzle curved wall), and spanwise (pointing toward the nozzle side wall) directions, respectively, with the origin located at the nozzle centerline at the throat. All of the spatial dimensions are in inches. The grid used with the wall matching functions (WMF) has about 3.9 million cells and will be referred to as the WMF grid. It should be noted that all simulations on the WMF grid also utilized turbulence models with the wall matching functions. The integrate-to-the-wall (ITW) grid has about 34.5 million cells, does not require the use of the wall matching functions, and will be referred to as the ITW grid. The grid densities near the centerline of the nozzle are similar for both grids in the cross-flow directions, with the ITW grid containing about twice as many points in the streamwise direction. The resulting values of dimensionless wall distance $(\mathrm{y}+)$ of the first off-the-wall cell-center, obtained for the thermally perfect simulations at the high limit 

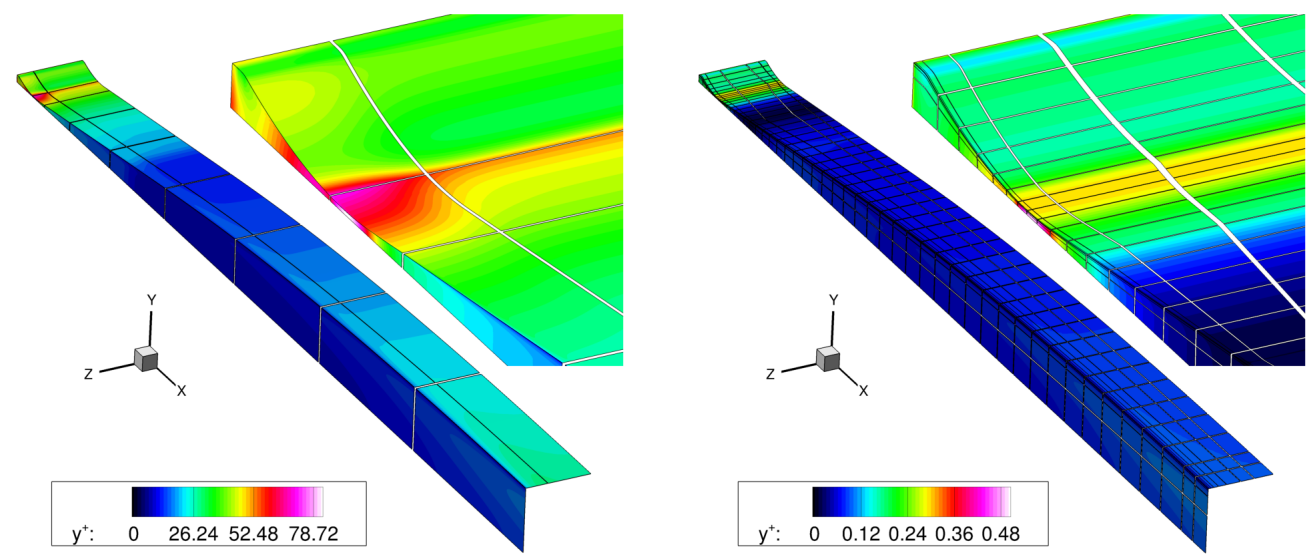

Figure 6. Contours of the dimensionless wall distance $(y+)$, obtained for the thermally perfect simulations at the high limit of total temperature (left) with wall matching functions (WMF), and (right) integrate-to-the-wall (ITW). The inset shows a close-up of the nozzle throat section. Top-left quarter of the facility nozzle is shown.

of the total temperature are shown in Fig. 6. The results for the cases at the low limit of the total temperature are comparable. For simulations utilizing the wall functions, the $y+$ values ranged from about 10 to 80 , with an average of about 25, and the largest values located at the nozzle throat. The y+ values for the ITW grid were less than one everywhere. Figure 7 shows line plots of the Mach number, static temperature, and static and pitot pressures at the nozzle centerline vs. axial distance obtained from the CFD solutions with WMF (lines) and ITW (symbols) grid. The inset on each figure provides focus on the data near the nozzle exit plane. The changes in the centerline exit values as a function of near-wall modeling approach are relatively small with $0.1,0.3$, and 1.5 and $0.8 \%$ for the Mach number, static temperature, and static and pitot pressures, respectively. Figure 8 shows contour plots of the Mach number, static temperature, and static and pitot pressures at the nozzle exit plane obtained from the CFD solutions with the WMF and ITW grids. It should be noted that for the static pressure contours the color scale has been focused on the exit plane such that even small differences are visible. Examination of the color scale values, however, reveals that the variation of the static pressures in the core flow is within $2 \%$. Overall, the differences are fairly small between these solutions, however, there is a slight thickening of the thermal boundary layers for simulations utilizing the WMF grid. This thickening does not seem to significantly impact the extent of the core flow for the quantities of interest. These simulations indicate that, for the current cases, the WMF models perform quite satisfactorily.

A grid sensitivity study was performed for simulations with the WMF grid for the thermally perfect case at the high limit of the total temperature. Only the WMF grid was used because, as shown above, the ITW solutions do not produce significantly different results. Figure 9 shows line plots of the Mach number, static temperature, and static and pitot pressures at the nozzle centerline vs. axial distance obtained from the CFD solutions on the coarse (lines) and fine (symbols) grids. The inset on each figure provides focus on the data near the nozzle exit plane. The changes in the centerline exit values as a function of grid density are $0.3,0.5$, and 2 and $1.4 \%$ for the Mach number, static temperature, and static and pitot pressures, respectively. Since, according to the Grid Convergence Index (GCI), ${ }^{22}$ for factor of two grid refinements, a conservative estimate of the numerical accuracy is conservatively proportional to the difference between coarse and fine grid solutions, we can expect that our numerical errors for the solutions on the fine grid are comparable to those reported above. Figure 10 shows contour plots of the Mach number, static temperature, and static and pitot pressures at the nozzle exit plane obtained from the CFD solutions on the coarse (C) and fine (F) grids. The differences are minimal between these solutions, however, there is a small thickening of the boundary layers on the coarse grid. This thickening is responsible for the reduction in the effective exit area and corresponding reduction in the exit Mach number for the coarse grid solution.

The sensitivity to the turbulence model was assessed by comparing the CFD simulations utilizing the baseline turbulent physics model of Menter ${ }^{20}$ to those with the explicit algebraic Reynolds-stress model (EARSM) of Rumsey and Gatski ${ }^{21}$ for the thermally perfect case, at the high limit of the total temperature. Figure 11 shows the line plot of the Mach number vs. axial distance, and Mach number contours at the nozzle exit plane for the CFD solutions obtained 

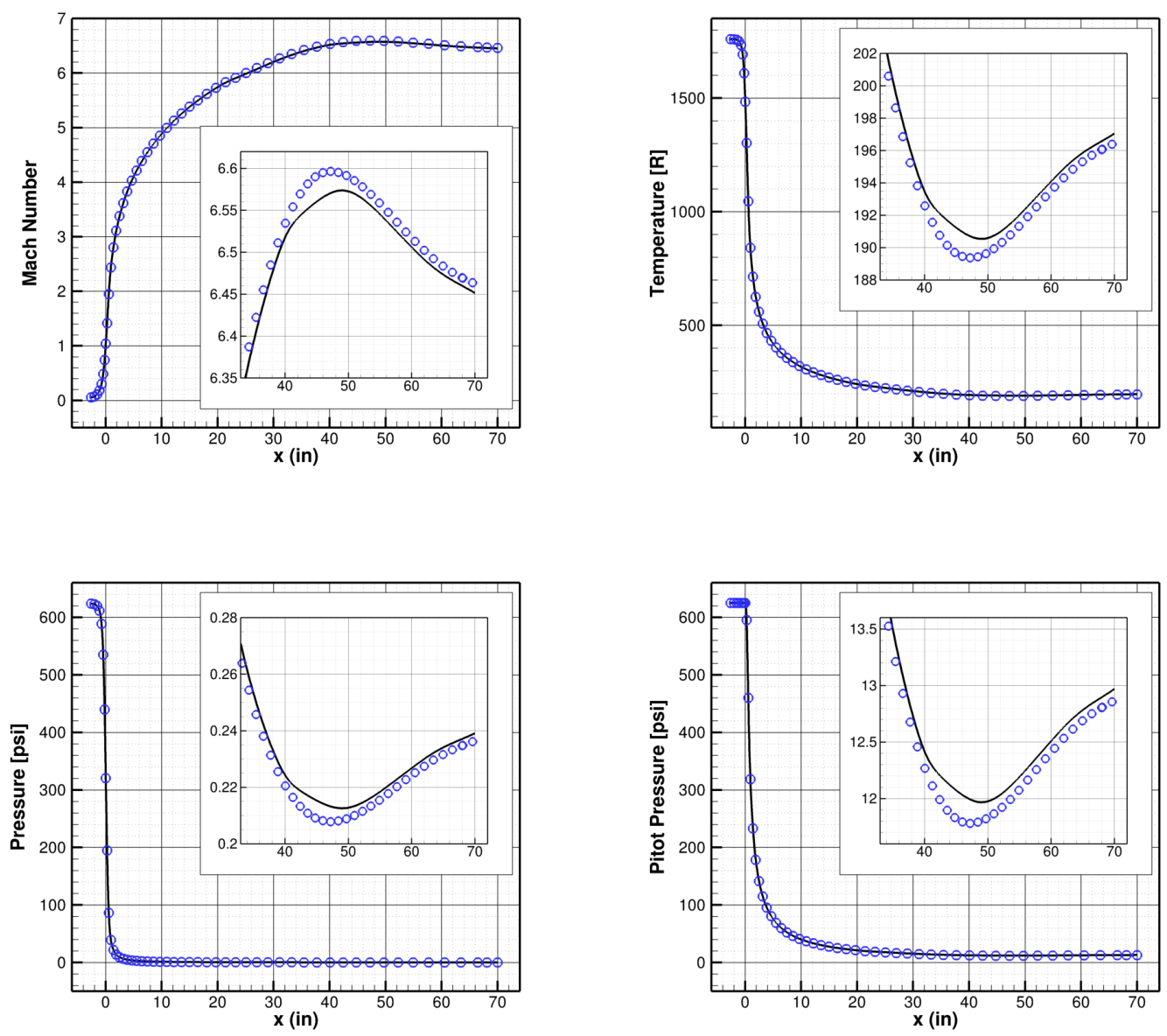

Figure 7. Line plots of the Mach number, static temperature, and static and pitot pressures at the nozzle centerline vs. axial distance obtained from CFD solutions with wall matching functions (WMF) (line), and integrate-to-the-wall (ITW) (symbols) grids. The inset on each plot provides a focus on the data near the nozzle exit plane. 

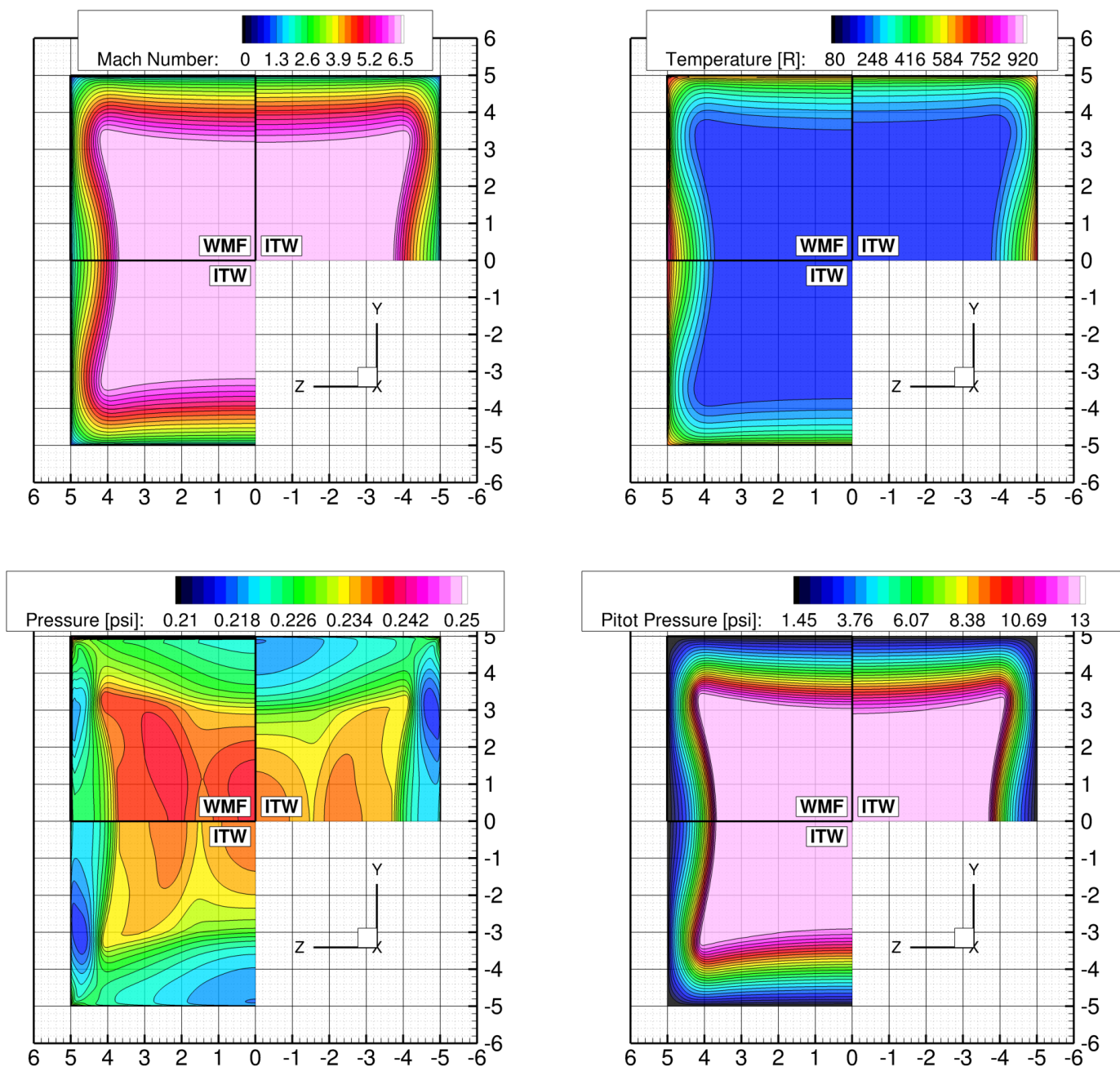

Figure 8. Contour plots of the Mach number, static temperature, and static and pitot pressures at the nozzle exit plane obtained from CFD solutions with wall matching functions (WMF), and integrate-to-the-wall (ITW) grids. 

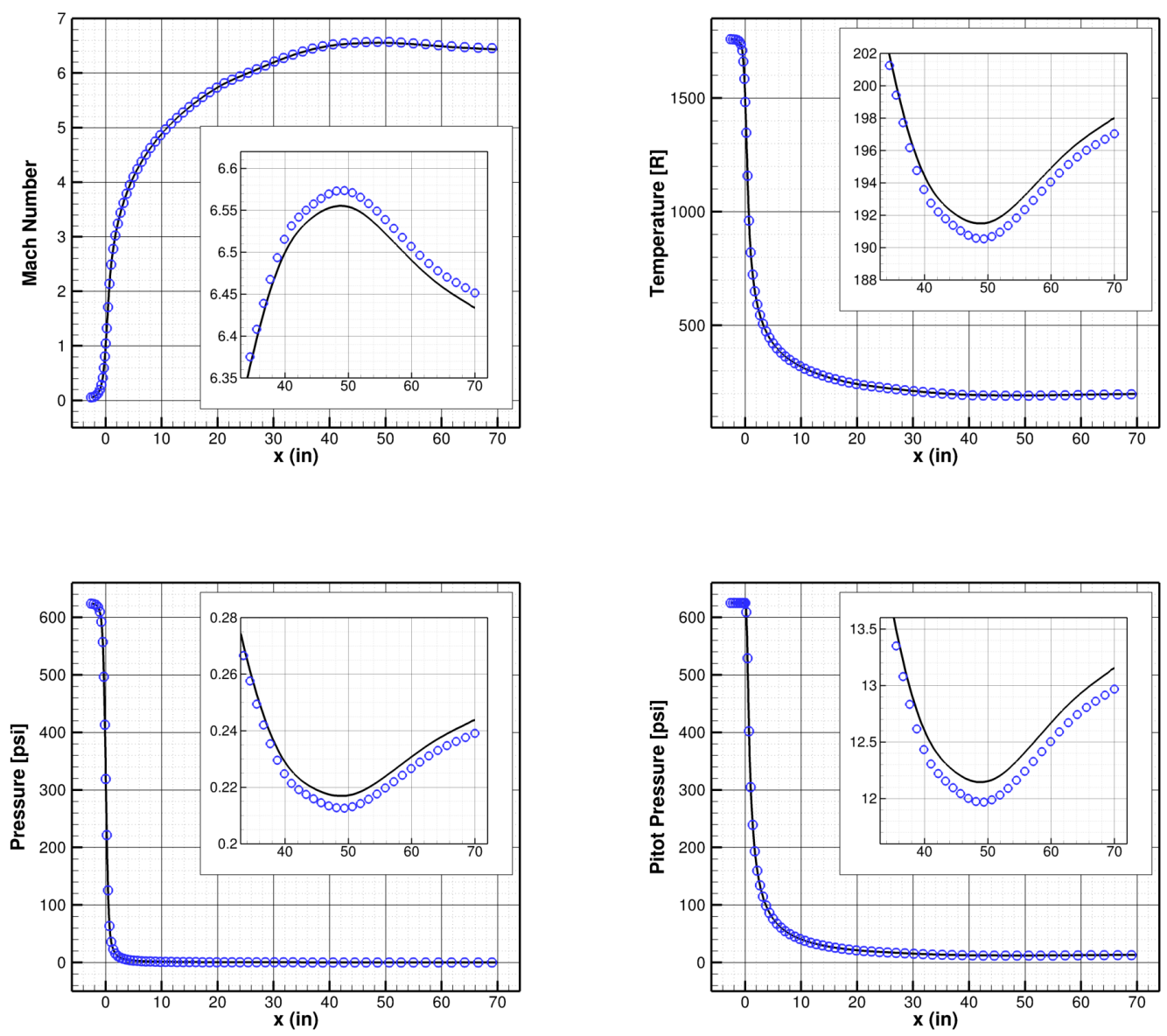

Figure 9. Line plots of the Mach number, static temperature, and static and pitot pressures at the nozzle centerline vs. axial distance obtained from CFD solutions on the coarse (line) and fine (symbols) grids. The inset on each plot provides a focus on the data near the nozzle exit plane. 

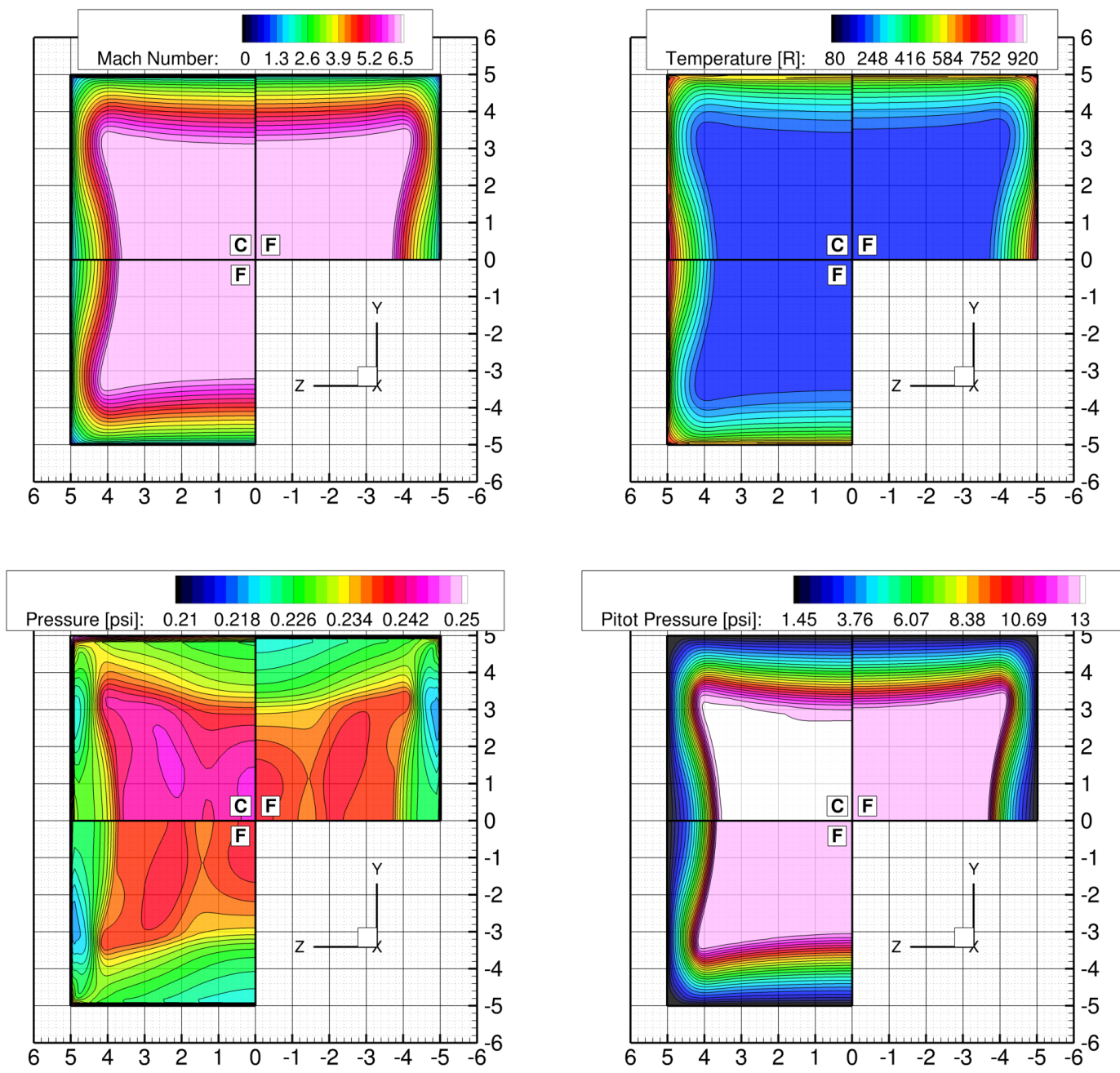

Figure 10. Contour plots of the Mach number, static temperature, and static and pitot pressures at the nozzle exit plane obtained from CFD solutions on the coarse (C) and fine (F) grids. 

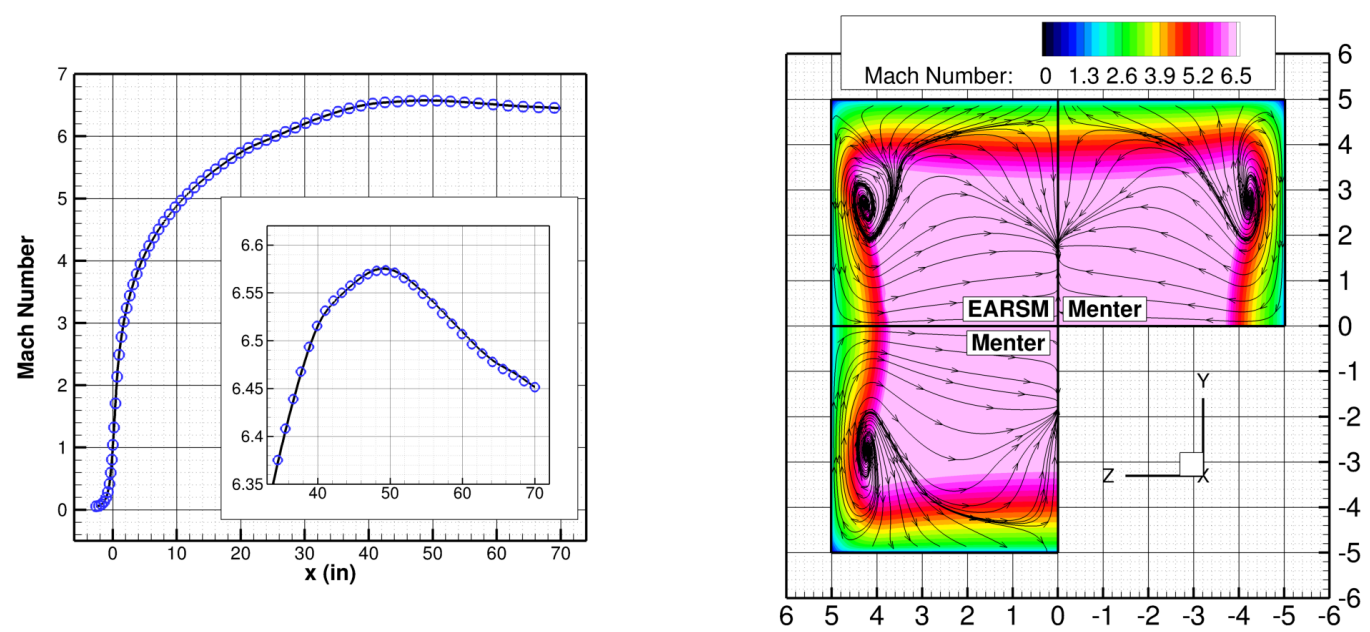

Figure 11. Line plot of the Mach number vs. axial distance (left) and contours of the Mach number with superimposed stream traces at the nozzle exit plane obtained from CFD solutions using the Menter baseline (lines) and explicit algebraic Reynolds-stress model (EARSM) (symbols) turbulence models.

using the two models. The velocity stream traces are also shown at the exit plane to highlight some of the detailed differences between the flow features. The presence of cross-flow vortical structures near the corners of the nozzle is driven solely by pressure gradients when the Menter linear eddy viscosity model is utilized. The EARSM non-linear model captures the combined effect of the pressure gradients and Reynolds stress anisotropies near the nozzle corners, which modifies the size and shape of the corner recirculation flow patterns. However, the boundary layer thicknesses for these two cases are nearly identical resulting in no significant change (less than $0.2 \%$ ) in the core flow exit Mach number and the corresponding properties.

The sensitivity to the thermal wall boundary conditions was assessed by comparing the CFD simulations utilizing a simplified $1 \mathrm{D}$ heat transfer model and an isothermal wall boundary conditions with the temperature set to $540^{\circ} \mathrm{R}$ for all walls for the same thermally perfect case, at the high limit of the total temperature, as above. Adiabatic wall conditions were not used because they were expected to significantly thicken the boundary layers, thereby introducing more flow blockage, which would lead to greater departures from the measured data as will be shown later. In contrast, the isothermal cold wall simulations are expected to reduce the boundary layer thickness, thereby bounding the analysis between our best guess for a thermal wall environment (i.e., the 1D heat transfer model) and a cold facility nozzle (i.e., isothermal walls). Figure 12 shows the wall temperatures on the top and side walls of the nozzle obtained with the 1D heat transfer model, and the contour plots of the Mach number and static temperature at the nozzle exit plane. The wall temperatures for the case utilizing the 1D heat transfer boundary condition are all higher than for the isothermal case. The measured integrated heat loss to the walls is greater for the latter by about $25 \%$. The aerodynamic boundary layer, visible via the Mach number isolines, and the core flow Mach number, seem unaffected by the wall thermal boundary conditions. The thermal boundary layer, visualized via the isolines of the static temperature, is slightly thinner for the isothermal wall case, which is consistent with the increased cooling at the wall. Similar to the comparisons between the turbulence models, the resulting differences between core flow Mach number and other static properties obtained from these two simulations are less than $0.2 \%$.

The impact of the thermodynamic nonequilibrium modeling is assessed by comparing CFD simulations utilizing the thermally perfect model to those with the explicit modeling of thermodynamic nonequilibrium for a case at the high limit of the total temperature. Only the high limit of the total temperature is investigated because the thermodynamic nonequilibrium effects are expected to be the most pronounced for this case, thereby bounding the impact for the current simulations. A two-temperature model, with the temperatures corresponding to the equilibrated translationalrotational, and vibrational-electronic energy modes, respectively, is used here. ${ }^{6,17}$ In addition, because the nozzle inflow plane in the current simulations is located upstream of the facility nozzle throat (near the nozzle plenum), the nozzle inflow conditions were assumed to be in thermodynamic equilibrium. Therefore, any thermodynamic nonequi- 

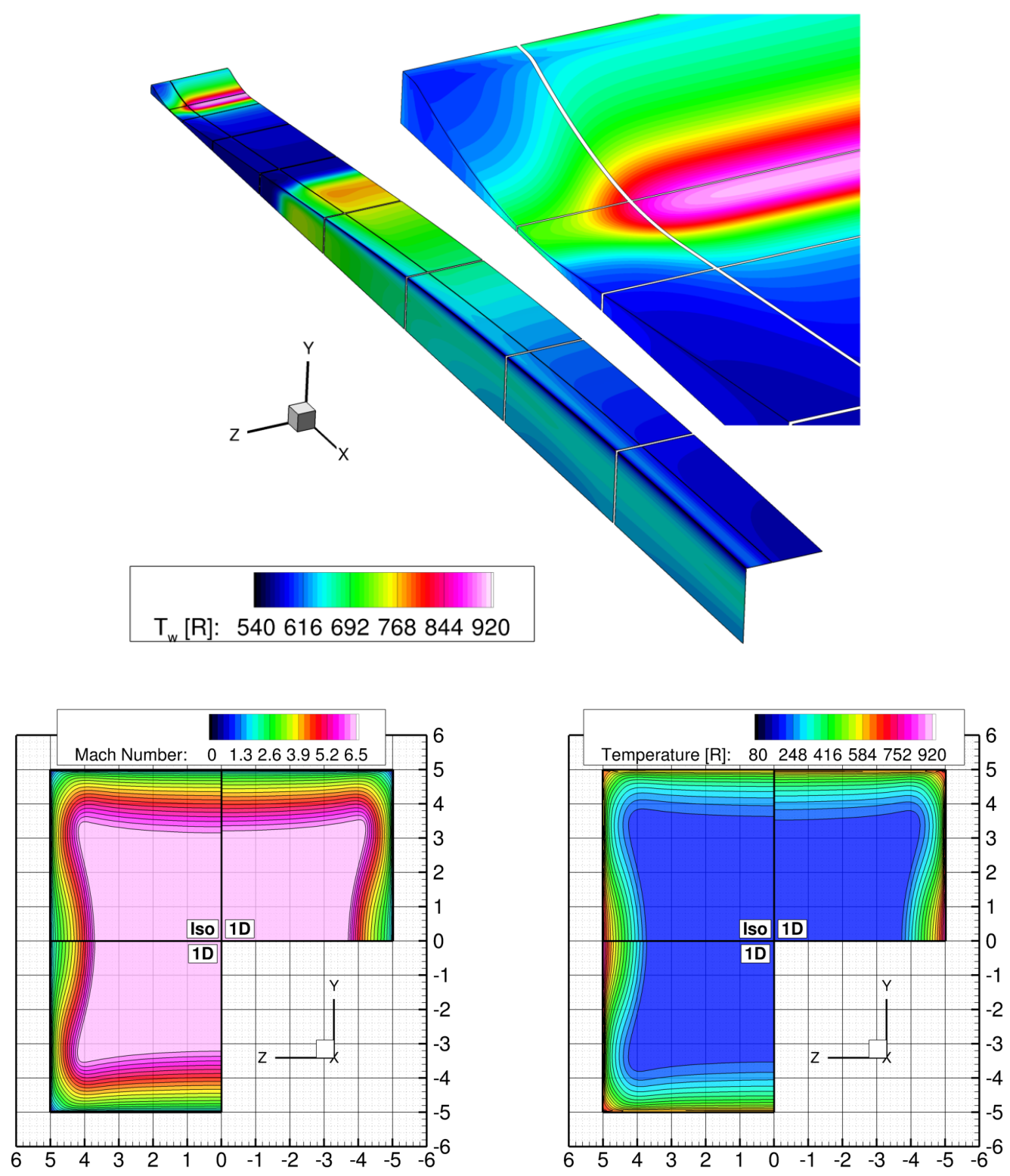

Figure 12. Contours of the wall temperature for the CFD solutions utilizing the 1D heat transfer thermal wall boundary conditions (top), and contours of the Mach number and static temperature at the nozzle exit plane obtained from CFD solutions using 1D heat transfer model (1D) and isothermal (Iso) conditions. 
librium effects are only a result of rapidly accelerating the flow through the converging-diverging nozzle. Figure 13 shows line plots of the Mach number, static temperatures, and static and pitot pressures at the nozzle centerline vs. axial distance obtained from the CFD solutions using thermal equilibrium (black lines) and nonequilibrium (blue symbols) models. The Mach number for the thermal nonequilibrium case is computed using the conventional definition of the speed of sound, with the ratio of specific heats computed using, and the static temperature corresponding to, the translational-rotational temperature. For brevity, the static temperature here implies the thermodynamically equilibrated, and the translational-rotational values, for the thermal equilibrium, and nonequilibrium simulations, respectively. The red symbols on the temperature plot denote the vibrational temperature with vibrational freezing occurring just downstream of the nozzle throat. The inset on each figure provides focus on the data near the nozzle exit plane. It should be noted that the Mach number, the static temperatures, and static pressure corresponding to the equilibrium and nonequilibrium simulations account for different physics, and therefore should be compared cautiously, primarily to qualitatively assess the impact of thermodynamic nonequilibrium modeling under the current conditions of interest. For the equilibrium simulations, the Mach number, the static temperature, and static pressure implicitly include the contributions of equilibrated vibrational energy modes through the specific heat polynomials, whereas for the nonequilibrium simulations, only translational-rotational energy is accounted for by these variables. Although, based on gas dynamic considerations, the static pressure depends only on the translational-rotational energy modes, ${ }^{23}$ its value is still impacted (lowered) by the thermal nonequilibrium via the lower value of the translational-rotational temperature appearing in the ideal-gas equation of state used to compute it. This lower value of the static pressure may still be partially offset by the changes in the flow density between these cases. The pitot pressure is computed assuming the flow equilibrates as it stagnates, thus this quantity can be quantitatively compared between these cases. The differences between the thermal equilibrium and nonequilibrium cases for the Mach, static temperature, and static and pitot pressures are $1.2,5,3$, and $0.3 \%$, respectively. It is important to note, that although the thermodynamic nonequilibrium model noticeably impacts some properties at the exit of the nozzle, the quantities that could be measured experimentally, that is, pitot pressure, and the total temperature, are only weakly, and not at all, respectively, impacted. Although, the static pressure shows a moderate level of impact, the issues associated with selecting a flow-dependent recovery factor for the static pressure probe measurements and the sensitivities to even small probe angle-of-attack or flow angularity, prevent reliable (to within $4 \%{ }^{12}$ ) measurement of the static pressure in the core flow of the nozzle. However, if the static pressure could be accurately measured, then it could serve as an indicator of the existence of thermodynamic nonequilibrium in the core flow, as long as its value proved to be lower than that obtained from the equilibrium CFD. Figure 14 shows contour plots of the Mach number, static temperature, and static and pitot pressures at the nozzle exit plane obtained from the CFD solutions using thermal equilibrium (Th.Eq.) and nonequilibrium (Th.NEq.) models. The same differences, as those reported above, are visible in the Mach, static temperature, and static pressure values, and almost no difference in the pitot pressure values. The reason for this is that the flow thermally equilibrates during the stagnation process after passing through the normal shock standing ahead of the pitot pressure probe. As the flow equilibrates, the energy that was "frozen" in the vibrational modes of the facility air molecules, equilibrates with the the translational-rotational energy modes, thereby raising the static pressure and temperature to near their thermally perfect values. The differences that remain are a result of different total pressure losses through the normal shock for a thermally equilibrated vs. nonequilibrated nozzle core flow. Since the thermal nonequilibrium model reduces the energy in the translational-rotational modes, the static pressure upstream of the normal shock is lower than that for a corresponding equilibrated flow, causing a higher static pressure ratio and somewhat greater total pressure losses across the normal shock.

In summary, the sensitivity studies presented above show weak dependence of less than about $1 \%$ in the pitot pressure of the CFD solutions on the wall modeling approach (WMF vs. ITW), turbulence model used (Menter vs. EARSM), and the thermal wall boundary modeling (1D heat transfer vs. isothermal wall). However, the grid density (coarse vs. fine), and the thermodynamic nonequilibrium modeling both indicated a somewhat larger cumulative impact on the simulations results. Therefore, the comparisons with the experimental data that follow use the CFD simulations utilizing the WMF grid with the fine density, the Menter baseline turbulence model, 1D heat transfer thermal wall boundary conditions, and the thermodynamic nonequilibrium model.

\section{Results and Comparison with Experimental Data}

Figure 15 shows the contours of the Mach number, total pressure, and total temperature obtained from the CFD simulations utilizing the WMF grid with the baseline Menter turbulence model, 1D heat transfer model for the wall temperature boundary conditions, and the thermodynamic nonequilibrium model for the facility air. The left and 

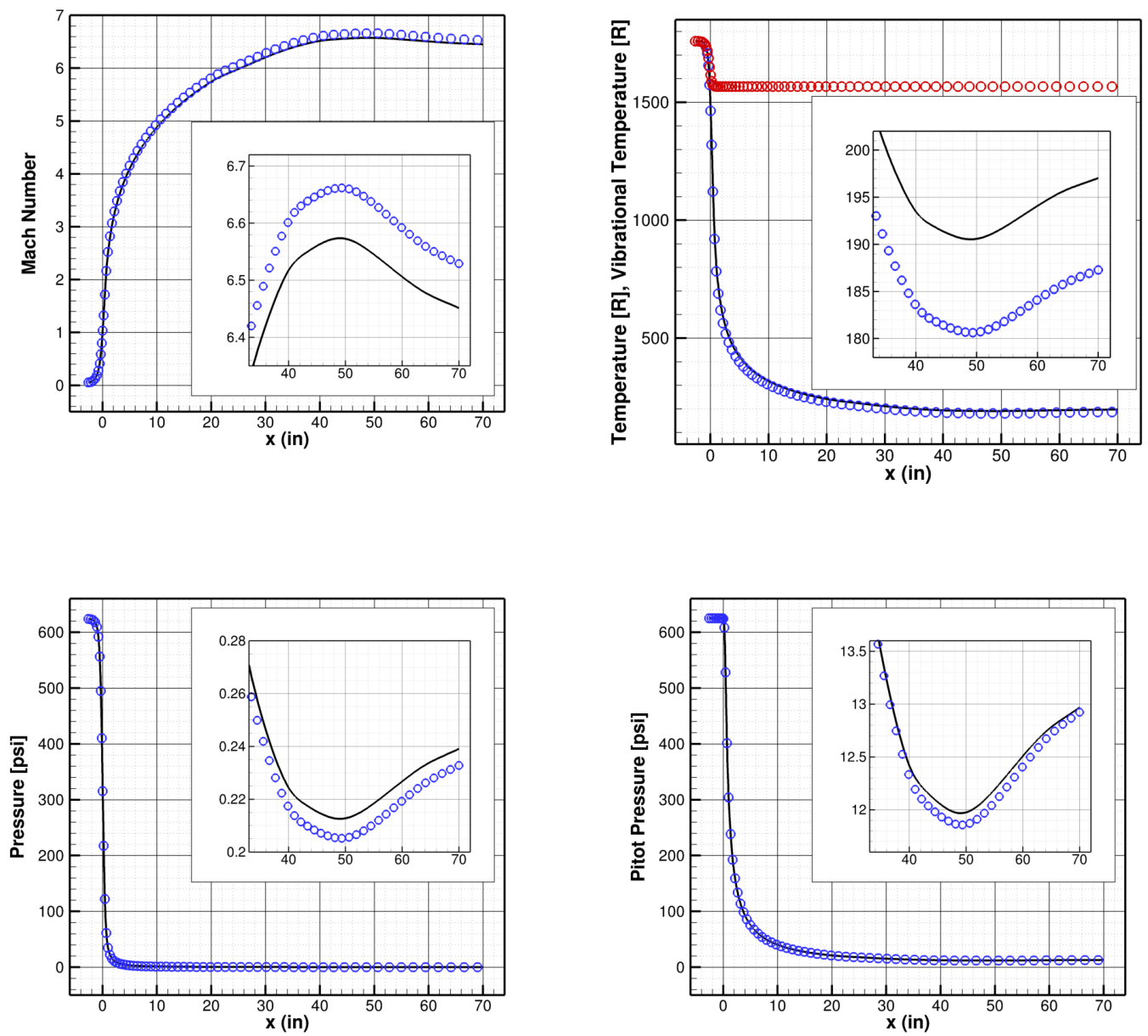

Figure 13. Line plots of the Mach number, static temperatures, and static and pitot pressures at the nozzle centerline vs. axial distance obtained from CFD solutions using thermal equilibrium (line) and nonequilibrium (symbols) models. The vibrational temperature for the nonequilibrium model is denoted by red symbols. The inset on each plot provides a focus on the data near the nozzle exit plane. 

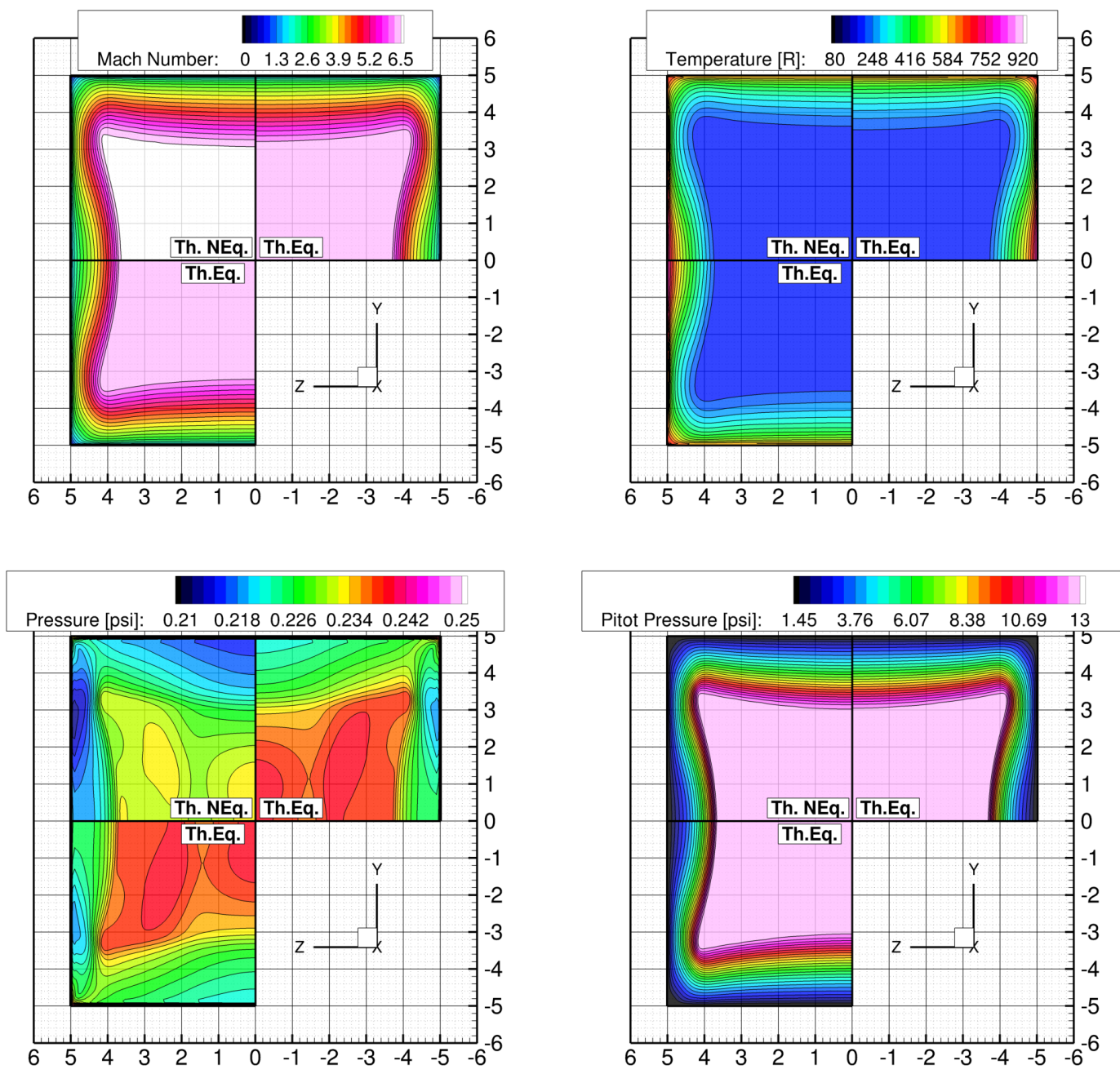

Figure 14. Contour plots of the Mach number, static temperature, and static and pitot pressures at the nozzle exit plane obtained from CFD solutions using thermal equilibrium (Th.Eq.) and nonequilibrium (Th.NEq.) models. 

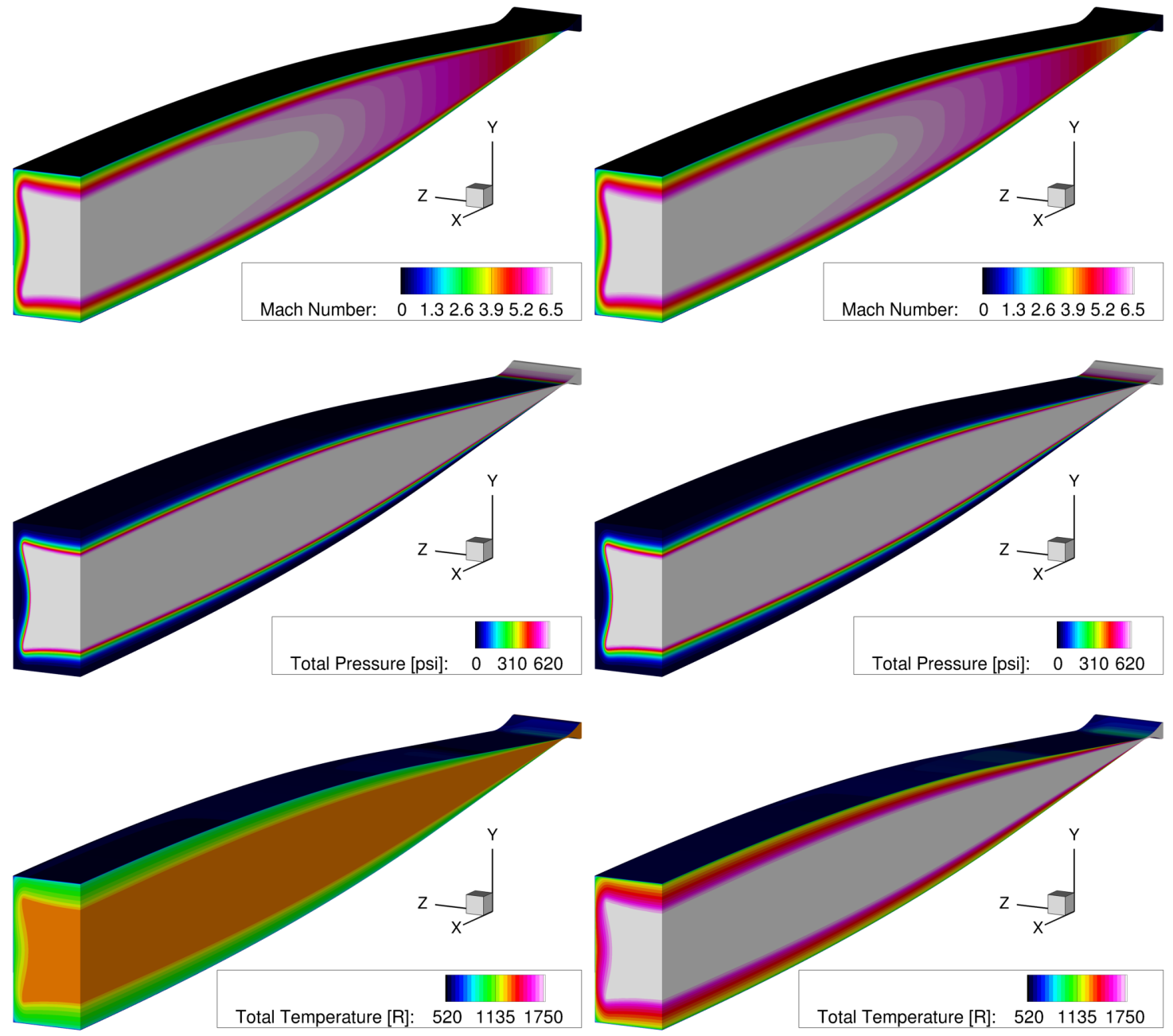

Figure 15. Contours of the Mach number, total pressure, and total temperature, obtained using CFD of the facility nozzle at the low (left column) and high (right column) values of the total temperature.

right columns correspond to the low and high total temperature cases, respectively. As a reminder, the Mach number in these plots is computed based on the translational-rotational values of both the ratio of specific heats and the temperature. Visual inspection reveals the flow fields corresponding to the low and high total temperature cases are qualitatively very similar. The flow passing through the facility nozzle, not only expands, but also develops relatively thick boundary layers (about 1.5, and 2.0 inches on the side and top/bottom walls, respectively). Surprisingly, given the off-design operation, no uncancelled waves are notable in either of the cases, and the boundary layers are comparable to those found during the on-design operation. ${ }^{4}$ Figure 16 shows contours of the Mach number; static, total, and pitot pressures; and total temperature at the exit plane of the nozzle comparing the low $(\mathrm{L})$ and high $(\mathrm{H})$ total temperature cases. A slight thickening of about $0.125-0.25$ inches of both the aerodynamic and thermal boundary layers are noted for the high total temperature cases. These boundary layers displace the exit core flow, and therefore reduce the effective area ratio of the nozzle. The facility nozzle core flow conditions obtained from the CFD simulations for both the low and high total temperature cases in thermal nonequilibrium are shown in Table 2. For comparison and reference, the corresponding values obtained from the thermal equilibrium cases are also shown. The values of the total pressure and total temperature are those corresponding to the nominal plenum values. The values of the static density, static pressure, pitot pressure, translation-rotational and vibrational temperatures, axial velocity, and the Mach number are obtained by averaging the core flow ( $\mathrm{y}, \mathrm{z}= \pm 2.5,3$ inches) at the nozzle exit plane. The smaller core flow height was chosen for the y-direction because the boundary layers are thicker on the curved, top and bottom, 

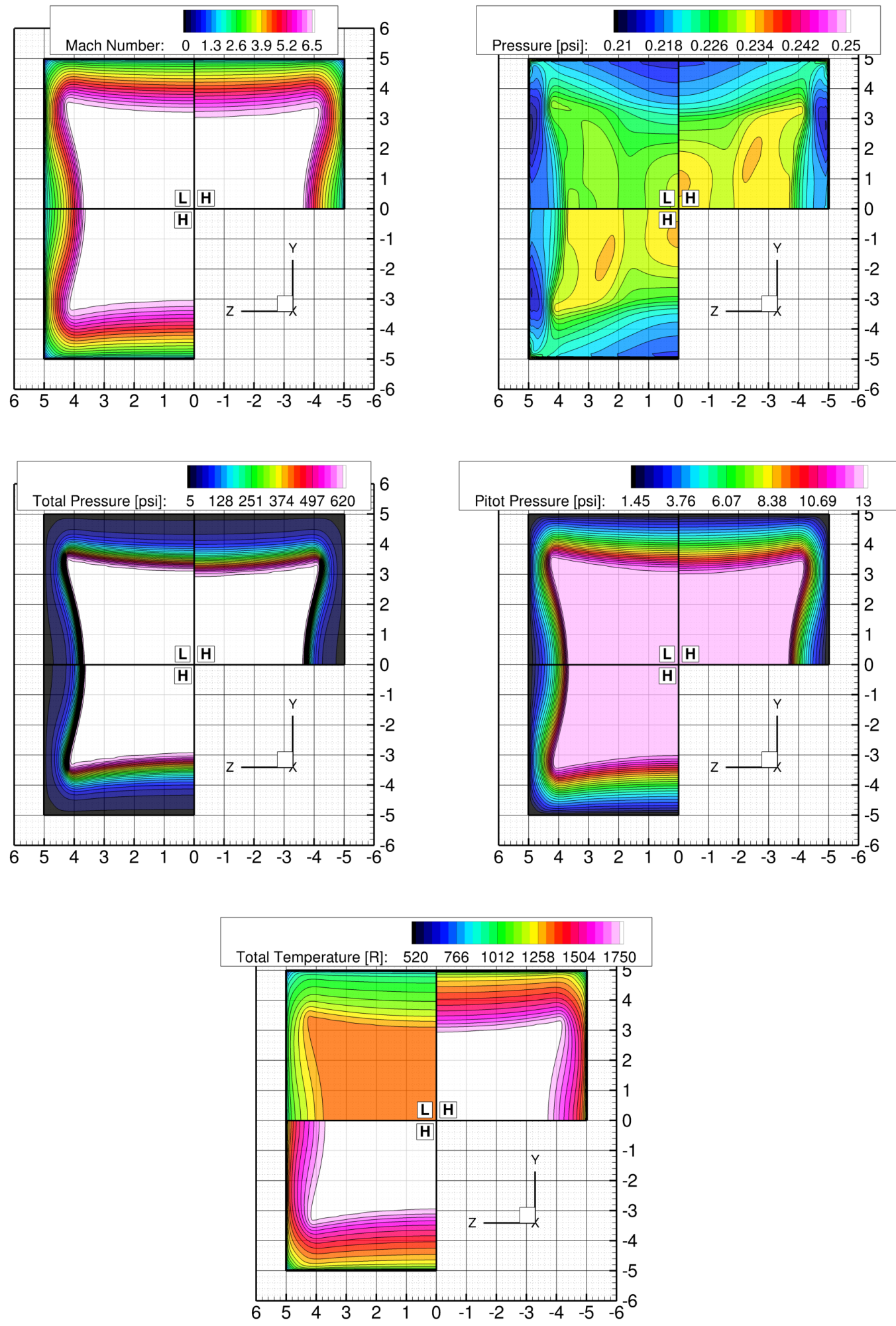

Figure 16. Contours of the Mach number; static, total, and pitot pressures; and total temperature at the nozzle exit plane obtained using CFD of the facility nozzle for the low $(\mathrm{L})$ and high $(\mathrm{H})$ values of the total temperature. 
Table 2. Exit core flow conditions obtained or computed from CFD.

\begin{tabular}{|c|c|c|c|c|}
\hline \multirow[b]{2}{*}{ Property } & \multicolumn{2}{|c|}{ Nonequilibrium } & \multicolumn{2}{|c|}{ Equilibrium } \\
\hline & Low Tot. Temp. & High Tot. Temp. & Low Tot. Temp. & High Tot. Temp. \\
\hline Total Pressure $^{\dagger}$ (psia) & 625 & 625 & 625 & 625 \\
\hline Total Temperature ${ }^{\dagger}\left({ }^{\circ} \mathrm{R}\right)$ & 1315 & 1760 & 1315 & 1760 \\
\hline Density $^{\ddagger}\left(\mathrm{lbm} / \mathrm{ft}^{3} \times 10^{-3}\right)$ & 4.471 & 3.331 & 4.409 & 3.256 \\
\hline Pressure $^{\ddagger}$ (psia) & 0.2291 & 0.2316 & 0.2320 & 0.2383 \\
\hline pitot Pressure & 12.77 & 12.88 & 12.81 & 12.94 \\
\hline Temperature ${ }^{\ddagger}\left({ }^{\circ} \mathrm{R}\right)$ & $137.8^{\S} / 1232.6^{\top}$ & $187.0^{\S} / 1566.6^{\top}$ & 141.5 & 196.9 \\
\hline $\mathrm{U}^{\ddagger}(\mathrm{ft} / \mathrm{s})$ & 3777 & 4388 & 3806 & 4447 \\
\hline $\operatorname{Mach}^{\ddagger}$ & $6.55^{\S}$ & $6.53^{\S}$ & 6.52 & 6.45 \\
\hline $\mathrm{Re} / \mathrm{L}^{\|}\left(1 /\right.$ in $\left.\times 10^{3}\right)$ & 295 & 203 & 287 & 194 \\
\hline Effective Area Ratio** & 78.1 & 77.9 & 77.9 & 77.5 \\
\hline Effective Exit Height $^{\dagger \dagger}$ (in) & 8.76 & 8.75 & 8.75 & 8.73 \\
\hline
\end{tabular}

\footnotetext{
$\dagger^{\dagger}$ nominal

$\ddagger$ average over nozzle core flow

$\S$ translational-rotational

I vibrational

$\|_{\text {computed using average values }}$

** computed using, total pressure, total temperature, pitot pressure (assuming equilibrium normal shock jump), and mass flow rate

${ }^{\dagger \dagger}$ computed using Effective Area Ratio and assuming square exit area
}

walls of the nozzle. The unit Reynolds number is computed using the average quantities, with the mixture viscosity evaluated using the equilibrium or the translational-rotational temperatures. The effective area ratio is obtained by using average values of the total pressure, total temperature, pitot pressure and the mass flow rate, together with the assumption of an equilibrium normal shock jump, and iterating a thermally perfect mixture to obtain the nozzle exit static properties, as well as, the effective exit area. The computed nozzle effective exit area is smaller than the actual area due to the boundary layer growth leading to the effective nozzle area ratio of about 78 , which reduces the core flow Mach number from about 6.9 for ideal expansion (see Table 1) to about 6.5 under the current conditions. The effective exit height is computed by assuming a square nozzle exit, indicating that the displacement thickness is about 0.63 inches. Furthermore, visual inspection reveals that the clean core flow region, suitable for free-jet experimentation downstream, extends plus or minus about 3 inches from the nozzle centerline in the cross-stream and spanwise directions.

The comparisons between the experimental data and the CFD are shown in Figs. 17, 18, and 19. The experimental and CFD data are denoted by symbols and lines, respectively. All data are time-averaged, as described previously, and labeled by run numbers. On Figs. 17, and 18, the black circles and magenta triangles denote data corresponding to the top and bottom or right $(\mathrm{z}=-5)$ and left $(\mathrm{z}=+5)$ walls of the nozzle, respectively. On Fig. 19, the different symbols (and colors) denote the spanwise (z-direction) position of the rake probe as indicated in the figure legends.

Figure 17 shows the static wall pressure data for the top/bottom and side walls for cases corresponding to the low (left column) and high (right column) total temperatures. As a reminder, the nozzle throat is located at the $\mathrm{x}=0$ position. The CFD agrees well with the data with some discrepancies in the early expansion portion of the nozzle at around $\mathrm{x}=20$ inches. Figure 18 also shows the static wall pressure data for the top/bottom and side walls for cases corresponding to the low (left column) and high (right column) total temperatures. However, these data are measured around the perimeter, near the nozzle exit plane at $x=68.45$ inches. The variation in the experimental data is representative of run-to-run variability in the average values of the static pressures. The maximum run-to-run variations in these data are about $0.01-0.02$ psi or about 5-10\%. This level of variability is much larger than what would be expected from the hardware measurement uncertainty considerations, and is thus far unexplained. There also appears to be a slight offset between the data collected on the top and bottom, and right and left walls. This could be an indication of some asymmetry in the nozzle flow character and/or formation of weak waves. The CFD data appears to mostly cut through the experimental measurements giving some confidence in the numerical modeling used here. The largest discrepancy between the data and the CFD occurs on the side walls for the high total temperature case.

Figure 19 shows the profiles of the pitot pressure, static pressure, and total temperature, measured using rake 

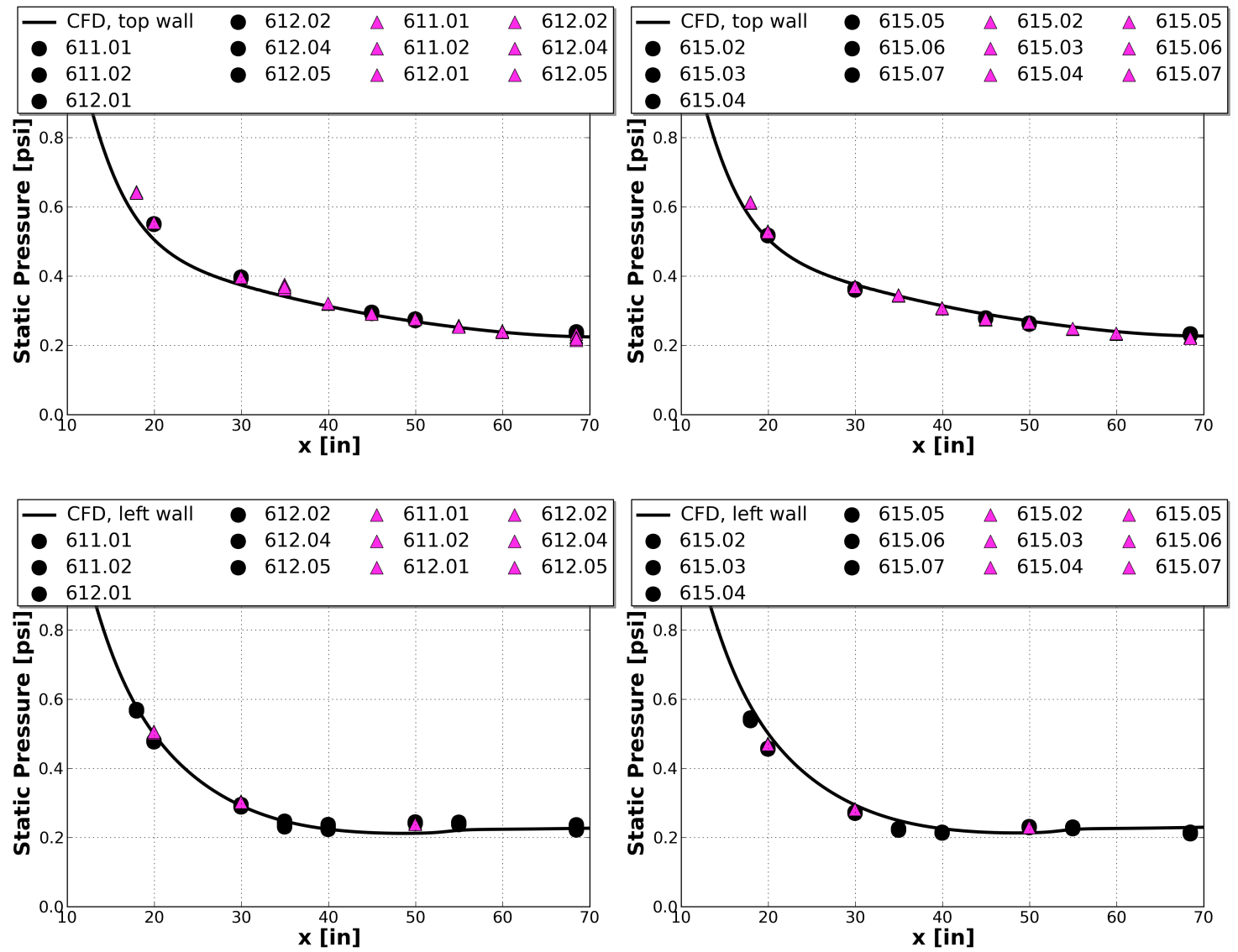

Figure 17. Profiles of the static pressure vs. streamwise distance along the top and side walls of the facility nozzle. Left and right columns correspond to the data for the low and high enthalpy experiments, respectively. Black circles and magenta triangles denote the data obtained on the top and bottom, and right and left walls, respectively. 

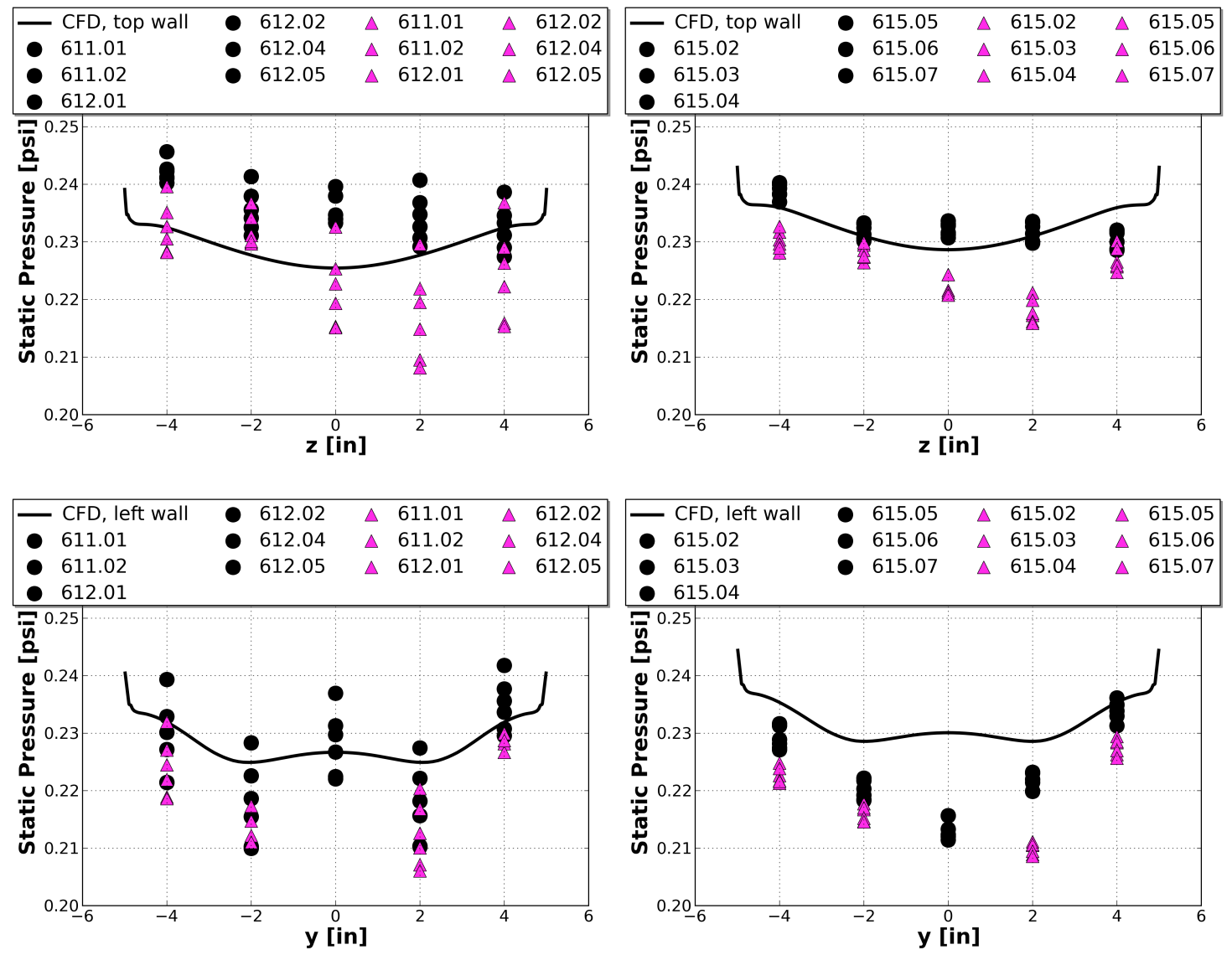

Figure 18. Profiles of the static pressure vs. cross-stream or spanwise distance along the top and side walls of the facility nozzle at a streamwise distance of 68.45 inches. Left and right columns correspond to the data for the low and high enthalpy experiments, respectively. Black circles and magenta triangles denote the data obtained on the top and bottom, and right and left walls, respectively. 
probes and obtained from the CFD, versus the cross-stream distance. Both the centerline and off-centerline spanwise locations are plotted. As before, the left and right columns correspond to low and high total temperature cases, respectively. The core flow values of the pitot pressure are overpredicted by the CFD data by 3 , and 5\%, for low and high total temperature cases, respectively. It should be noted that the grid dependence (coarse vs. fine) and nearwall modeling (WMF vs. ITW) studies discussed in the previous section revealed that about $1-2 \%$ of this difference could be attributed to the sensitivity of the solutions to the current grid density (see Fig. 9) and the choice of the wall modeling (see Fig. 7). The remainder of the difference is likely a combination of factors, with the most likely culprits being the misprediction of the boundary layer thickness, a weak shock wave system unpredicted by the CFD, and/or underestimation of the effects of the thermodynamic nonequilibrium.

The boundary layer thickness impacts the pitot pressure by aerodynamically "blocking" a portion of the nozzle outflow area, thereby changing the effective nozzle area ratio, the core flow Mach number, and other properties. When the boundary layer is thick the blockage is high, and the nozzle area ratio decreases, decreasing the core flow Mach number with it. In turn, the lower Mach number core flow experiences lower total pressure loss across the normal shock standing upstream of the pitot pressure probe, therefore increasing the pitot pressure measurement value. The boundary layer thickness, in general, is a function of the flow Reynolds number and the thermal wall boundary conditions, but could also be affected by the accuracy of the turbulence model used in the simulations. However, for the current case, the turbulent boundary layer prediction, with the exception of the details of the corner flows, is almost completely unchanged for two fundamentally distinct models (see Fig. 11). This is not to say that the boundary layer thickness is well predicted, instead only that the choice of the model does not seem to significantly impact the results. The accuracy of the models is a separate issue, which has been well documented, for example, for zero-pressure-gradient wall-bounded flows indicating good predictive capability. However, since by definition, no model is perfect, we can not rule out that both turbulence models slightly over predict the boundary layer growth rate under the current favorable-pressure-gradient conditions. An estimate of the level of misprediction of the boundary layer induced blockage may be obtained by recomputing the effective area ratio and exit height, as in Table 2, while substituting the core-average experimental values of the pitot pressure for the average pitot pressures from the CFD. The resulting effective area ratio and exit height are 80.2 and 8.88 inches, and 82.2 and 8.99 inches, for the low and high total temperature cases, respectively. The corresponding estimate for the displacement thickness is 0.56 , and 0.50 inches, respectively. Comparing these results to those found in Table 2, suggests that the displacement thickness is overpredicted in the CFD by about 0.07 and 0.13 inches, or about $14 \%$ and $26 \%$, respectively.

The formation of a weak shock wave system could also decrease the values of the measured pitot pressures by reducing the total pressure of the core flow approaching the exit plane. Furthermore, such a wave system would necessarily cross through the exit plane, causing a variation in the pitot pressure profiles through the core flow. A small decrease in the value of the measured pitot pressure is indeed noticeable near the centerline of the nozzle, as if a weak shock crossed between the centerline and the adjacent pitot probe, for both low and high total temperature cases. However, no such wave system formed in any of the CFD simulations. Nevertheless, it should be noted that the CFD did not consider any of the geometrical imperfections that could be present in the as-built geometry or that could form as a result of hot operation. As described earlier, the Mach 6 facility nozzle is constructed from two sections: the water-cooled copper throat section, and an uncooled stainless-steel expansion section. The interface between these parts occurs at 12.5 inches downstream of the nozzle throat. It is possible that a step exists at this location, which is unmodeled in the CFD, and could be the source of the wave system responsible for the decrease in the measured pitot pressure near the centerline.

The sensitivity of the pitot pressure to the thermodynamic nonequilibrium modeling was examined in Fig. 13. Although, the impact on the pitot pressure was determined to be only a fraction of one percent, the observed trend was in the right direction, that is, thermodynamic nonequilibrium effects lowered the value of the measured pitot pressure as compared to a case with a thermally perfect flow. However, the current nonequilibrium modeling assumes that the flow in the nozzle plenum is in thermal equilibrium, and the flow is well mixed and does not contain any thermal strata. These assumptions are reasonable given high pressures and long residence times in the nozzle plenum but not easily verifiable. And, the observation that the measured static pressures are lower than those predicted by the CFD gives some credence to the notion that the predicted nonequilibrium effects could be underestimated. The level of this underprediction might still be small because the predicted core flow static pressure values would decrease together with the decreasing pitot pressure in response to the decreasing boundary layer thickness.

The discrepancy between the total temperature measurements and the CFD is about $4 \%$, and $2 \%$ for the low and high total temperature cases, respectively. Because the total temperature values are not impacted by shocks, expansions, or thermal nonequilibrium effects, these values should more closely match the CFD. However, since the 

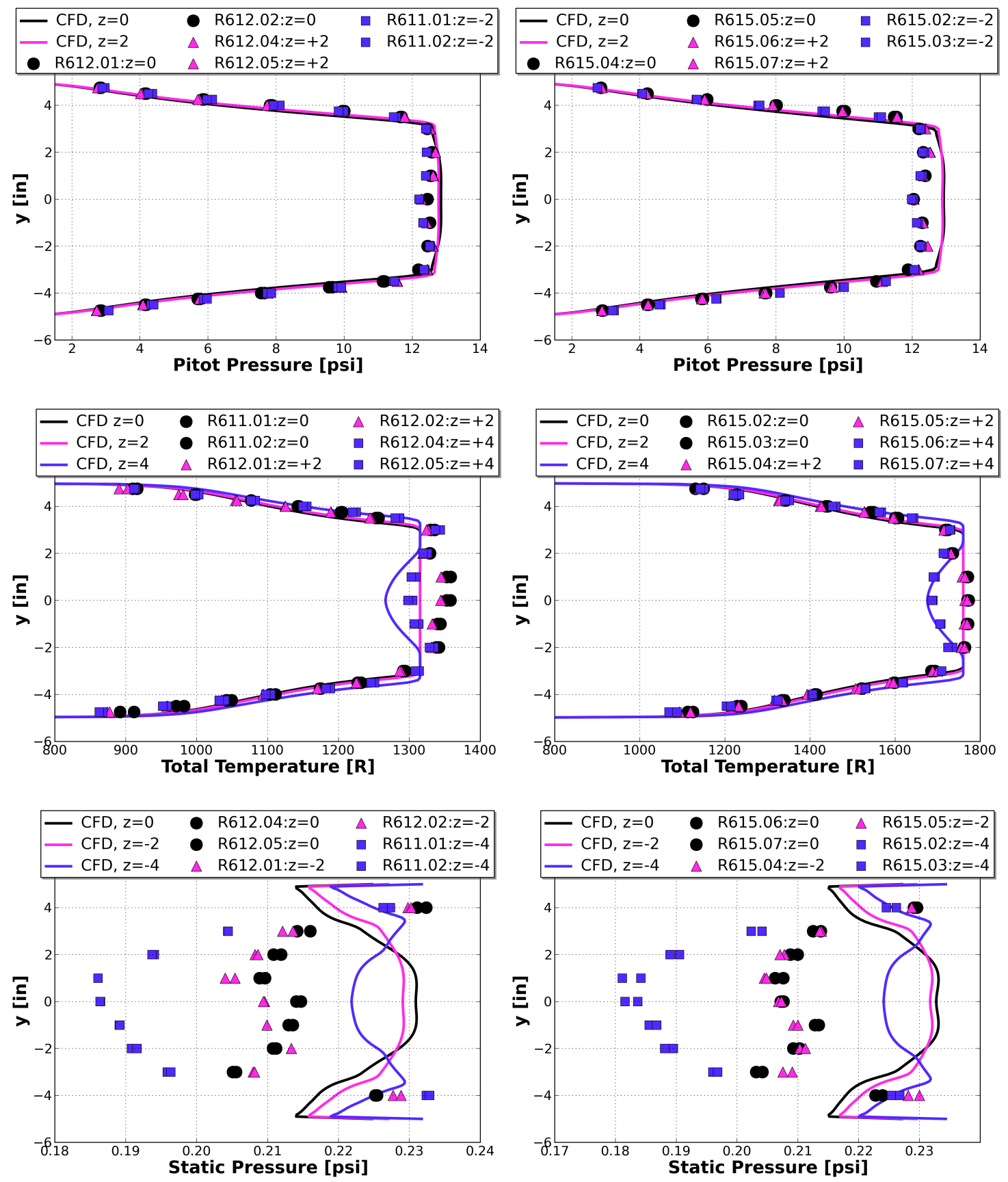

Figure 19. Profiles of the pitot pressure, static pressure, and total temperature obtained using rake probes at the nozzle exit plane for several experimental runs and compared to the CFD data. Left and right columns correspond to the data for the low and high total temperature experiments, respectively. 
nominal measure of the total temperature used for normalizing the experimental data is based on the sonic throat calculation, ${ }^{9}$ which assumes thermally equilibrated, one dimensional, well mixed, flow through the nozzle throat, there is a potential for a mismatch between the calculated and measured total temperature when the core flow is only partially thermally mixed. The total temperature measurement at $\mathrm{z}=4$, shows a decrease at the centerline because at that location the measurement rake has entered the side-wall boundary layer (see Fig. 16) where the total temperature decreases toward the cooled walls. This profile feature appears in both the measurements and the CFD indicating a reasonable prediction of the extent of the thermal boundary layers, although any mismatch here will be very sensitive to the accuracy of CFD prediction of the side-wall boundary layer thickness and rake position.

In-stream static pressure is overpredicted by the CFD by about $10 \%$ in the core flow and over $20 \%$ for centerline values at the $\mathrm{z}=4$ off-centerline spanwise location. This seems quite inaccurate, but several factors should be kept in perspective. First, recall that the static pressure probe values are "corrected" using a recovery factor obtained from the surrogate CFD simulations of the static pressure probe in the core flow under the comparable flow conditions (see Fig. 5 and discussion). For a given probe geometry, the recovery factor changes as a function of the free stream flow conditions, primarily the ratio of specific heats and the Mach number, which vary little in the core flow but change in the boundary layers. In practice, it is impractical to perform static probe CFD simulations for every condition needed, and instead few representative conditions are chosen for which probe CFD is performed and those results are then interpolated (or extrapolated) to other flow conditions as desired. In the current experiments, recall that all of the core flow static pressures are scaled by the same factor of 1.29, which was obtained from static probe CFD simulations for high total temperature conditions and using thermal equilibrium assumptions. A second factor of 1.08 , corresponding to a flow with a Mach number of 4.7, is used only for the top- and bottom-most static pressure probes, which reside well into the boundary layers. Note that the data at $\mathrm{z}=4$ location is within the side-wall boundary layer (see Fig. 16). At this location, the effective probe-local "free-stream" conditions are significantly different from those in the core flow, therefore the recovery factor will vary along the y-dimension. Without properly calibrating the probe recovery factors probe-by-probe, this data can only be treated in a qualitative manner. Therefore, the errors of $20 \%$ reported above for this data can not be used as an indication of deficiencies in the current simulations. Second, the potential misprediction of the boundary layer thickness discussed above for the pitot pressure measurement has an equivalent impact on the static pressure. That is, a misprediction in the exit core flow area that results in overprediction of the pitot pressure will also result in an overprediction of the static pressure. Because these quantities are related, under the current core flow conditions it can be shown that a $1 \%$ change in the pitot pressure produces about $1.5 \%$ change in the static pressure. Therefore, a 5\% overprediction in the pitot pressure is responsible for about $7.5 \%$ overprediction in the static pressure. This amount already accounts for the three quarters (7.5\% out of $10 \%)$ of the discrepancy between the experiments and the CFD. A portion (about 2\%) of the remainder can be attributed to the grid resolution sensitivity of the static pressure (see Fig. 9). Furthermore, unlike the pitot pressure, the static pressure measurements are much more sensitive to the level of thermodynamic nonequilibrium (see Fig. 13). Therefore, some portion of the remainder of the error could indicate a deficiency in the thermodynamic nonequilibrium models, a notion made more possible given that the unmodeled wave system discussed above, would serve to increase the core flow static pressures obtained from the CFD. Unfortunately, unlike the pitot and total temperature measurements, the sensitivity of the static pressure probes measurements to the probe's installed angle-of-attack and/or flow angularity presents a comparable level of uncertainty, as previously reported by Pinckney ${ }^{12}$, making it impossible to distinguish between measurement, and simulation and modeling errors to within about $4 \%$.

\section{Conclusions}

Computational fluid dynamics (CFD) analysis of the Mach 6 facility nozzle used in the EIMP experiments at the NASA Langley Research Center is presented. The CFD utilizes the VULCAN-CFD solver to perform threedimensional viscous RAS. The sensitivity of the CFD results to various numerical and modeling assumptions is also presented. Specifically, comparisons between results obtained with wall modeling and integrate-to-the-wall approaches, as well as, two distinctly different turbulence models, two thermal boundary conditions approaches, and thermal equilibrium and nonequilibrium models, are shown. These results reveal that current simulations are largely insensitive (observed less than $1 \%$ change in the pitot pressure) to the choice of the wall modeling approach, turbulence model, and thermal boundary conditions. Some sensitivity was found with respect to the thermal nonequilibrium model. Therefore, the simulations utilizing the less computationally expensive approach with wall modeling via the Wilcox wall matching functions, and the thermal two-temperature nonequilibrium model were chosen to compare with the experimental data. The experimental data consists of wall static pressure measurements along the nozzle top, 
bottom, and side walls, and around the perimeter near the nozzle exit plane. Additionally, in-stream rakes, positioned just downstream of the nozzle exit plane, provide measurements of the static and pitot pressures, and the total temperature at on- and off-centerline locations. The pitot and total temperature measurements agree with the CFD data to within about $5 \%$. The static pressure data measured by the probes agrees with the CFD to within about $10 \%$. These discrepancies are attributed to a combination of factors: for CFD, an apparent overprediction of the boundary layer growth rate leading to the increased aerodynamic blockage at the nozzle exit plane; possibility of a wave system that anchors at the unmodeled small step at the interface of the throat and expansion sections of the nozzle; and possible (although less likely) underestimation of the levels of the thermal nonequilibrium effects; for the total temperature measurements, thermal unmixedness of the core flow leading to a violation of assumptions inherent in the sonic throat method computations, as well as, inflow boundary conditions uncertainty; and finally, for the static pressure probe measurements, inherent uncertainty in the measurement due to the probe angle-of-attack and flow angularity. The reported level of agreement likely represents the state-of-the-art for the numerical and experimental approach considered in this work. Future work will further investigate the possibility of presence of shock and expansion wave system throughout the nozzle core flow. These compressible flow features have the potential to cause the nonuniformities observed near the nozzle centerline in the in-stream measurements, especially the pitot pressure.

\section{Acknowledgments}

This work is supported by the Hypersonic Technology Project in the NASA Aeronautics Research Mission Directorate (ARMD). Computational resources are provided by the NASA Langley Research Center and the NASA Advanced Supercomputing (NAS) Division.

\section{References}

\footnotetext{
${ }^{1}$ Guy, R.W., Torrence, M.G., Sabol, A.P., and Mueller, J.N., "Operating Characteristics of the Langley Mach 7 Scramjet Test Facility,” Tech. Rep. TM-81929, NASA, 1981.

${ }^{2}$ Thomas, S. and Guy, R., "Expanded Operational Capabilities of the Langley Mach 7 Scramjet Test Facility," Tech. Rep. TP-2186, NASA, 1983.

${ }^{3}$ Guy, R.W., Rogers, R.C., Puster, R.L., Rock, K.E., and Diskin, G.S., "The NASA Langley Scramjet Test Complex," in 32nd ASME, SAE, and ASEE, Joint Propulsion Conference and Exhibit, Lake Buena Vista, FL, 1996.

${ }^{4}$ Gaffney, R. and Korte, J., "Analysis and Design of Rectangular-Cross-Section Nozzles for Scramjet Engine Testing," in 42nd AIAA Aerospace Sciences Meeting and Exhibit, 2004-1137, Reno, NV, 2004.

${ }^{5}$ Cabell, K., Drozda, T.G., Axdahl, E.L., and Danehy, P.M., "The Enhanced Injection and Mixing Project at NASA Langley," in JANNAF 46th CS / 34th APS / 34th EPSS / 28th PSHS Joint Subcommittee Meeting, Albuquerque, NM, 2014.

${ }^{6}$ Baurle, R., "Analysis of Facility Non-Equilibrium Thermodynamic Effects on HIFiRE Ground Tests," in JANNAF 46th CS / 34th APS / 34th EPSS / 28th PSHS Joint Subcommittee Meeting, Albuquerque, NM, 2014.

${ }^{7}$ Drozda, T.G., Axdahl, E.L., and Cabell, K.F., "Pre-Test CFD for the Design and Execution of the Enhanced Injection and Mixing Project at NASA Langley Research Center," in JANNAF 46th CS / 34th APS / 34th EPSS / 28th PSHS Joint Subcommittee Meeting, Albuquerque, NM, 2014.

${ }^{8}$ Cabell, K.F. and Rock, K.E., "A Finite Rate Chemical Analysis of Nitric Oxide Flow Contamination Effects on Scramjet Performance," Tech. Rep. TP-212159, NASA, 2003.

${ }^{9}$ Winovich, W., "On the Equilibrium Sonic-Flow Method For Evaluating Electric-Arc Air-Heater Performance," NASA/TN D-2132, Ames Research Center, Moffett Field, CA, 1964.

${ }^{10}$ Wilcox, D.C., Turbulence Modeling for CFD, DCW Industries, Inc., La Cañada, CA, 2000.

${ }^{11}$ Witte, D.W., Irby, R.G., Auslander, A.H., and Rock, K.E., "1998 Calibration of the Mach 4.7 and Mach 6 Arc-Heated Scrmajet Test Facility Nozzles,” NASA/TM 2004-213250, NASA Langley Research Center, Hampton, VA, 2004.

${ }^{12}$ Pinckney, S.Z., “A Short Static-Pressure Probe Design For Supersonic Flow,” NASA/TN D-7978, NASA Langley Research Center, Hampton, VA, 1975.

${ }^{13}$ VULCAN-CFD, “http://vulcan-cfd.larc.nasa.gov/," 2016.

${ }^{14}$ van Leer, B., "Towards the Ultimate Conservative Difference Scheme. V: A Second-Order Sequel to Godunov's Method," J. Comput. Phys., Vol. 32, No. 1, 1979, pp. 101-136. 659.

${ }^{15}$ Edwards, J.R., "A Low-Diffusion Flux-Splitting Scheme for Navier-Stokes Calculations," Comput. Fluids., Vol. 26, No. 6, 1997, pp. 635-

${ }^{16}$ McBride, B.J., Gordon, S., and Reno, M.A., "Thermodynamic Data for Fifty Reference Elements," NASA Technical Paper 3287/REV1, NASA, Cleveland, OH, 2001.

${ }^{17}$ Gnoffo, P., Gupta, R.N., and Shinn, J.L., "Conservation Equations and Physical Modes for Hypersonic Air Flows in Thermal and Chemical Nonequilibrium,” NASA Technical Paper 2867, NASA, Hampton, VA, 1989.

${ }^{18}$ Krist, S.L., Biedron, R.T., and Rumsey, C.L., "CFL3D User's Manual (Version 5.0),” NASA Technical Report TM-1998-208444, NASA, Hampton, VA, 1998.
} 
${ }^{19}$ Litton, D.K., Edwards, J.R., and White, J.A., "Algorithmic Enhancements to the VULCAN Navier-Stokes Solver," in 16th AIAA Computational Fluid Dynamics Conference, AIAA 2003-3979, Orlando, FL, 2003.

${ }^{20}$ Menter, F.R., "Two-Equation Eddy-Viscosity Turbulence Models for Engineering Applications," AIAA J., Vol. 32, No. 8, 1994, pp. 15981605.

${ }^{21}$ Rumsey, C.L. and Gatski, T.B., "Summary of EASM Turbulence Models in CFL3D with Validation Test Cases," NASA/TM 2003-212431, NASA Langley Research Center, Hampton, VA, 2003.

${ }^{22}$ Roache, P.J., Verification and Validation in Computational Science and Engineering, Hermosa Publishers, 1998.

${ }^{23}$ Vincenti, W.G. and Kruger, C.H.J., Introduction to Physical Gas Dynamics, Krieger Publishing Co., Malabar, FL, 1965. 